\title{
Characterization of the plasma membrane proteins and receptor-like kinases associated with secondary vascular differentiation in poplar
}

\author{
Dongliang Song $\cdot$ Wang Xi $\cdot$ Junhui Shen $\cdot$ \\ Ting Bi $\cdot$ Laigeng $\mathbf{L i}$
}

Received: 14 December 2010/ Accepted: 14 March 2011/Published online: 24 March 2011

(C) The Author(s) 2011. This article is published with open access at Springerlink.com

\begin{abstract}
The constituents of plasma membrane proteins, particularly the integral membrane proteins, are closely associated with the differentiation of plant cells. Secondary vascular differentiation, which gives rise to the increase in plant stem diameter, is the key process by which the volume of the plant body grows. However, little is known about the plasma membrane proteins that specifically function in the vascular differentiation process. Proteomic analysis of the membrane proteins in poplar differentiating secondary vascular tissues led to the identification 226 integral proteins in differentiating xylem and phloem tissues. A majority of the integral proteins identified were receptors (55 proteins), transporters (34 proteins), cell wall formation related (27 proteins) or intracellular trafficking (17 proteins) proteins. Gene expression analysis in developing vascular cells further demonstrated that cambium differentiation involves the expression of a group of receptor kinases which mediate an array of signaling pathways during secondary vascular differentiation. This paper provides an outline of the protein composition of the plasma membrane in differentiating secondary vascular tissues and sheds light on the role of receptor kinases during secondary vascular development.
\end{abstract}

Electronic supplementary material The online version of this article (doi:10.1007/s11103-011-9771-3) contains supplementary material, which is available to authorized users.

D. Song · W. Xi · J. Shen · T. Bi · L. Li (ه)

Laboratory of Synthetic Biology, Institute of Plant Physiology and Ecology, Chinese Academy of Sciences, 300 Fenglin Rd, Shanghai 200032, China

e-mail: lgli@sibs.ac.cn
Keywords Plasma membrane protein . Integral protein - Receptor-like kinase . Proteomics · Vascular differentiation · Poplar

\section{Introduction}

Secondary vascular differentiation occurs mainly in angiosperm dicot and gymnosperm trees. Tree trunks grow in diameter through the activity of its vascular cambium, which is a secondary meristem that divides inwards to produce secondary xylem cells and outwards to develop secondary phloem cells. To date, many studies have profiled the global gene expression during secondary vascular differentiation in order to understand the molecular mechanisms underlying this secondary growth process. Gene transcripts profiled in poplar and other tree species during cell differentiation after vascular cambium division indicated that the differentiation is under stage-specific transcriptional regulation and that a number of genes are found to be expressed in association with the differentiation (Hertzberg et al. 2001; Allona et al. 1998; Pavy et al. 2008; Schrader et al. 2004). Meanwhile, proteomic profiling has also provided an outline of which genes are expressed during the various stages of secondary meristem cell differentiation in poplar. Regulatory proteins for cell cycle progression and cell fate were found to be expressed in the early stages while proteins for secondary wall formation were found predominantly in the later stages of differentiation (Du et al. 2006).

Secondary vascular tissue of tree species features several types of specialized cells including fiber cells, sieve and vessel elements, which are formed during the differentiation process after cambium cell division. Membrane proteins are believed to play important roles over the course of cell differentiation via various functions such as 
cell signaling, catalysis and cross-membrane transport (Tan et al. 2008). For the characterization of the plasma proteins related to plant cell wall formation, detergent-resistant plasma membrane microdomains was analyzed in aspen cell suspensions and found to contain a group of key carbohydrate synthases (Bessueille et al. 2009). Subcellular proteomic analysis was conducted for protein inventory of cell organelles such as mitochondria, chloroplast, and peroxisomes (Lilley and Dupree 2007; Baginsky 2009; Yu et al. 2008; Reiland et al. 2009). However, the particular protein constituents in the plasma membrane of secondary vascular tissues has been little studied. While this manuscript was in the process of being prepared, a study reported the detection of 956 proteins from the membrane preparation of Populus (Nilsson et al. 2010). Among them, transporter and receptor proteins were found to be major constituents of membrane proteins which displayed a pronounced distribution among leaf, xylem and phloem tissues. Leaf plasma membrane contained a high proportion of transporters, constituting almost half of the integral proteins while xylem plasma membranes contained an abundance of membrane trafficking proteins. Overall, those results demonstrated that membrane proteins are differentially distributed in the various tissues of poplar (Nilsson et al. 2010).

In plants, receptor-like kinases (RLKs) have been shown to be a crucial class of transmembrane proteins for the perception of various signals on the cell surface. It has been reported that signaling mediated by ligand-RLK pathways play an essential role in regulating cell-to-cell communication and cell differentiation during postembryonic development in plants (Fletcher et al. 1999; Lenhard and Laux 1999; Fukuda 2004; De Smet et al. 2009). Recently, a RLK has been studied for its implication in vascular cell differentiation. PXY/TDR (PHLOEM INTERCALATED WITH XYLEM/TRACHEARY ELEMENT DIFFERENTIATION INHIBITORY FACTOR RECEPTOR) has been reported as an important receptor-like kinase that controls the orientation of cell division during vascular development (Hirakawa et al. 2008; Fisher and Turner 2007). A peptide, TDIF (TRACHEARY ELEMENT DIFFERENTIATION INHIBITORY FACTOR), which is encoded by CLE41 and CLE44 in A. thaliana has been demonstrated as a ligand which binds specifically to TDR/PXY (Hirakawa et al. 2008). TDIF-PXY/TDR forms a ligand-receptor system involved in regulating vascular cell differentiation in Arabidopsis.

In the present paper, we present the proteomic profile of the plasma membrane isolated after a two-phase separation from the differentiating xylem and phloem tissues in poplar. More than 1,500 proteins were found to be associated with the plasma membrane isolation. Of those, a total of 226 proteins were identified as integral plasma membrane proteins. Overall, the results of the present study offer an independent categorization of the plasma membrane proteins isolated after a rigorous separation procedure. In particular, a group of RLKs were identified in the plasma membrane. Analysis of the cell-specific gene expression revealed that a group of the RLK genes were differentially expressed in a pattern which suggests that different RLKs may mediate different signaling pathways during secondary vascular development. Profiling of the expression of RLK genes provides a line of new information for dissecting how secondary vascular tissues are developed through serial signaling regulation on the plasma membrane.

\section{Materials and methods}

Plant materials and micro-dissection

A group of Populus female cloning trees (Populus $\times$ euramericana $c v$. 'Nanlin895'), which were grown in an experimental field with 3 years old, were used for collection of a large amount of tissue samples. The sample collection was carried out in the morning of May 11, 2008, when the leaves of the trees were fully developed. The upper part of tree stems was sectioned for tissue collection. After the stem bark was peeled, differentiating phloem and xylem were examined and harvested directly into liquid nitrogen and stored for later use.

The same clone of the tree, which was grown in a greenhouse was used for micro-dissection. Cell samples of the differentiating secondary vascular tissue were acquired from cross-sections of poplar stem by micro-dissection as described (Song et al. 2010). Vascular cambium, differentiating xylem and phloem, and cortex cells were collected from dissecting a total of 40 stem sections, which amounted to approximately 12,000 cells in each sample. Total transcript preparation from the sampled cells was as described (Song et al. 2010).

\section{Real-time quantitative PCR quantification} of cell-specific gene expression

For real-time quantitative PCR measurement, primers were designed to amplify a specific fragment (100-300 bp in length) of the detected genes. The primer specificity was confirmed by amplification of a single specific band. Measurements were performed on a MyiQ Real-Time PCR Detection System (Bio-Rad, Winston-Salem, NC, USA). The PCR reaction was carried out in a volume of $20 \mathrm{ul}$ containing $50 \mathrm{ng}$ of cDNA template using SYBR Green Master Mix (TOYOBO, Osaka, Japan). PCR program was: one cycle of $95^{\circ} \mathrm{C}$ for $2 \mathrm{~min}$, followed by 45 cycles of $95^{\circ} \mathrm{C}$ 
for $15 \mathrm{~s}, 58^{\circ} \mathrm{C}$ for $15 \mathrm{~s}$ and $72^{\circ} \mathrm{C}$ for $20 \mathrm{~s}$. After amplification, the PCR product was examined by measuring their melting curves to ensure the accuracy of the reaction. The abundance of the gene transcripts was normalized against Actin 2 expression. The expression scale of ST651, a characterized cytokinin receptor gene, was set to 1 , equivalent to 1/1000 of the Actin 2 transcript abundance, for the relative comparison of gene expression.

\section{Microsomal fraction preparation}

Differentiating xylem and phloem samples (100 g) were ground in liquid nitrogen together with $1 \%$ PVPP to fine powder, and then homogenized at $4^{\circ} \mathrm{C}$ in $500 \mathrm{ml}$ extraction buffer containing $0.5 \mathrm{M}$ Tris- $\mathrm{HCl}, \mathrm{pH} 8.5,0.7 \mathrm{M}$ sucrose, $0.1 \mathrm{M} \mathrm{KCl}, 50 \mathrm{mM}$ EDTA, $1 \mathrm{mM}$ PMSF, 2\% (v/v) $\beta$-mercaptoethanol, $1 \mathrm{mM}$ leupeptin and $1 \mathrm{mM}$ pepstatin (Saravanan and Rose 2004; Suzuki et al. 2006). Afterwards the homogenate was centrifuged at $10,000 \mathrm{~g}$ for $10 \mathrm{~min}$ at $4^{\circ} \mathrm{C}$ and filtered with Miracloth. The filtrate was diluted by an equal volume of ice cold water and centrifuged at $150,000 \mathrm{~g}$ for $30 \mathrm{~min}$ to collect the microsomal fraction. The pellet was then washed three times with ice cold water at $4^{\circ} \mathrm{C}$ to remove residual supernatant proteins.

\section{Plasma membrane separation and quality assay}

The plasma membrane fraction was separated from the above microsomal preparation using a dextran-PEG aqueous two phase system with minor modification(Tanaka et al. 2004). The microsomal fraction was suspended in $10 \mathrm{mM} \mathrm{KH}{ }_{2} \mathrm{PO}_{4} / \mathrm{K}_{2} \mathrm{HPO}_{4}$ buffer $(\mathrm{pH} 7.8)$ and then added to a partition system consisting of 6.3\% PEG3350, 6.3\% dextran T-500, $0.3 \mathrm{M}$ sucrose and $10 \mathrm{mM} \quad \mathrm{KH}_{2} \mathrm{PO}_{4} /$ $\mathrm{K}_{2} \mathrm{HPO}_{4}$ buffer (pH 7.8). After the first partition, the upper phase was recovered and partitioned with fresh lower phase twice. The final upper phase was diluted 1:5 with ice cold water. The plasma membrane pellet was collected by centrifugation at $150,000 \mathrm{~g}$ for $30 \mathrm{~min}$ at $4^{\circ} \mathrm{C}$.

The quality of the isolated plasma membrane was estimated by monitoring different types of $\mathrm{H}^{+}$-ATPase as indicated by $\mathrm{P}$ type $\mathrm{H}^{+}$-ATPase for plasma membrane, $\mathrm{F}$ type $\mathrm{H}^{+}$-ATPase for mitochondrion or chloroplast and $\mathrm{V}$ type $\mathrm{H}^{+}$-ATPase for vacuoles, respectively, (Sze 1985). ATPase activity was measured according to the methods described (Sandstrom et al. 1987; Tanaka et al. 2004) with modification. Suspended plasma membrane protein (about $5 \mu \mathrm{g}$ ) was added to assay buffer (50 mM Tris-Mes ( $\mathrm{pH} 6.5$ ), $5 \mathrm{mM} \mathrm{MgSO}_{4}$, $50 \mathrm{mM} \mathrm{KCl}, 5 \mathrm{mM}$ NaATP, $0.1 \mathrm{mM} \mathrm{Na} \mathrm{MoO}_{4}, 125 \mathrm{mM}$ sucrose, $0.0125 \%(\mathrm{w} / \mathrm{v})$ Triton $\times 100)$ with or without inhibitor (inhibitors: $100 \mu \mathrm{M} \mathrm{Na}_{3} \mathrm{VO}_{4}$ for P- $\mathrm{H}^{+}$-ATPase, $2 \mathrm{mM} \mathrm{NaN}_{3}$ for $\mathrm{F}-\mathrm{H}^{+}$-ATPase and $50 \mathrm{mM} \mathrm{KNO}_{3}$ for $\mathrm{V}-\mathrm{H}^{+}$-ATPase). Assay mixture was incubated at $37^{\circ} \mathrm{C}$ for $10 \mathrm{~min}$ and then terminated by adding stop solution containing $2 \% \mathrm{H}_{2} \mathrm{SO}_{4}, 5 \%$ SDS, $0.5 \% \mathrm{Na}_{2} \mathrm{MoO}_{4}$. After being stopped, $10 \%$ ascorbic acid was added into the reaction mixture and incubated for $10 \mathrm{~min}$ at room temperature, ATPase activity was determined spectrophotometrically at $A_{660}$.

For membrane protein identification, the plasma membrane pellet was dissolved in SDS buffer $(0.5 \mathrm{M}$ Tris- $\mathrm{HCl}$ $\mathrm{pH} 8.5,2 \%$ (v/v) $\beta$-mercaptoethanol, 30\% (v/v) glycerol, 4\%SDS, $1 \mathrm{mM}$ PMSF, $1 \mathrm{mM}$ leupeptin and $1 \mathrm{mM}$ pepstatin) and heated for $5 \mathrm{~min}$ at $80^{\circ} \mathrm{C}$, the dissolvent was subsequently centrifuged at $12,000 \mathrm{~g}$ for $30 \mathrm{~min}$ at room temperature to remove insoluble debris. Then the supernatant was extracted with an equal volume of water-saturated phenol. After the phenol phase was recovered, it was re-extracted three times with the microsomal extraction buffer. Then the membrane proteins were precipitated from the phenol phase by adding 5 volumes of cold methanol containing $0.1 \mathrm{M}$ ammonium acetate. After overnight precipitation at $-20^{\circ} \mathrm{C}$, the proteins were pelleted by centrifugation at $12,000 \mathrm{~g}$ for $10 \mathrm{~min}$ at $4^{\circ} \mathrm{C}$. The pellet was washed three times with $90 \%$ cold methanol, followed by another wash with $90 \%$ acetone. After drying under vacuum at $4{ }^{\circ} \mathrm{C}$, the pellet was resuspended in $500 \mu \mathrm{l}$ of rehydration buffer (7 M urea, $2 \mathrm{M}$ thiourea, 4\% w/v CHAPS, $40 \mathrm{mM} \mathrm{DTT,} 1 \%$ $\mathrm{v} / \mathrm{v}$ IPG buffer, $\mathrm{pH} 4-7$ ). After centrifugation at 20,000 $g$ for $30 \mathrm{~min}$ at $4{ }^{\circ} \mathrm{C}$, the plasma proteins were divided into two portions: one was soluble in the rehydration buffer and the other insoluble.

Protein separation and digestion

The above soluble portion of protein (about $1.2 \mathrm{mg}$ ) was applied to 2-DE analysis according to (Fiorani Celedon et al. 2007). After 2-DE separation, proteins were detected by silver or CBB G-250 staining method. Three protein extract replicates were performed and gel images were analyzed with the Image Master Platinum software (v. 6.0) (GE Healthcare, Amersham Bioscience). Meanwhile the insoluble portion of protein was analyzed by SDS-PAGE following our previous used protocol (Song et al. 2010). After the gels were stained, 46 protein bands were detected in each sample.

Protein samples excised from 2-DE spots or 1-DE bands were cut into $1 \mathrm{~mm}$ cubes. Samples were destained with $100 \mu \mathrm{l}$ of $50 \% \mathrm{v} / \mathrm{v}$ ACN/25 mM ammonium bicarbonate solution. The digestion was incubated with a $10 \mathrm{ng} / \mu \mathrm{l}$ of trypsin solution in $25 \mathrm{mM}$ ammonium bicarbonate at $37^{\circ} \mathrm{C}$ for $12 \mathrm{~h}$. The peptide mixtures were extracted twice with $8 \mu \mathrm{l}$ of $50 \% \mathrm{v} / \mathrm{v}$ ACN, $0.5 \% \mathrm{v} / \mathrm{v}$ formic acid (FA). The extracts were dried under protection of $\mathrm{N}_{2}$ and resuspended in $5 \% \mathrm{ACN}, 0.1 \% \mathrm{FA}$. The protein samples from 2-DE spots were analyzed by MALDI-TOF-MS/MS and the proteins from 1-DE bands were identified by nano-LC-MS/ MS analysis. 


\section{Protein identification by MALDI-TOF-MS/MS}

Digested proteins from the spot samples were redissolved in $50 \% \mathrm{ACN}, 0.1 \% \mathrm{TFA}$ and $5 \mathrm{mg} / \mathrm{ml} \mathrm{CHCA}$. Then the samples were spotted on a target plate. The MALDI-TOFMS was performed on an ABI 4700 Proteomics Analyzer (Applied Biosystems, Framingham, MA, USA) instrument, followed by MS/MS analysis. Mass spectra were obtained on a mass range of 700-3,200 Da using a laser beam $(335 \mathrm{~nm}, 200 \mathrm{~Hz})$. The instrument was performed in a positive ion mode using an acceleration voltage of $20 \mathrm{kV}$. The mass spectra were acquired by the data-dependent acquisition method with 5-6 of the strongest precursor ions selected for MS/MS analysis. Myoglobin digested by trypsin was used to calibrate the mass instrument. MALDITOF-MS/MS data were analyzed using MASCOT (Matrix Science, London) search software against Populus tricocarpa protein database (http://genome.jgi-psf.org/Poptr1_1/ Poptr1_1.download.ftp.html) assuming the digestion enzyme trypsin and the search parameters for MASCOT as described (Zhang et al. 2010).

\section{Protein analysis by Nano-flow LC-MS/MS}

Analyses of the digested proteins from band samples were performed on a LC-20AD system (Shimadzu, Tokoya, Japan) connected to an LTQ Orbitrap mass spectrometer (ThermoFisher, San jose, CA, USA) as described (Song et al. 2010). Tandem mass spectra were extracted by BioWorks version 3.3.1 sp1 (ThermoFisher). All MS/MS samples were analyzed using Sequest (ThermoFisher, version 28). The parameters for the Sequest were: peptide tolerance, $50 \mathrm{ppm}$; MS/MS tolerance, 1.0 Da. Peptide identifications were accepted only if they could be established at greater than $95.0 \%$ probability as specified by the Peptide Prophet algorithm (Keller et al. 2002). Protein probabilities were assigned by the Protein Prophet algorithm (Nesvizhskii et al. 2003).

\section{Results}

Preparation of the plasma membrane proteins

from differentiating xylem and phloem

According to microscopic analysis, cambium cells, which are usually restricted to only 2-4 layers of cells, were found to be attached to both differentiating phloem and xylem tissue when the bark was peeled from the poplar stem (Fig. 1). This is different from previous report in eucalyptus, in which cambium cells are found to be stuck only to the side of the phloem (Fiorani Celedon et al. 2007). Thus, both differentiating phloem and xylem tissues collected for our study actually contained vascular cambium cells and were used for protein isolation.

To study the plasma membrane protein constituents of differentiating vascular tissues, the collected tissue was first isolated for the crude microsomal fraction, from which the plasma membrane fraction was then purified using an aqueous two phase partition system (Schindler and Nothwang 2006). To ensure the high quality of the preparation, the purified plasma membrane was examined for the membrane-specific marker activities. $\mathrm{H}^{+}$-ATPase is widely used as a specific marker for distinguishing between subcellular membranes. Vanadate-sensitive $\mathrm{P}$ type $\mathrm{H}^{+}$-ATPase is found in plasma membrane while azidesensitive $\mathrm{F}$ type $\mathrm{H}^{+}$-ATPase is specific to mitochondrion and chloroplast, and nitrate-sensitive $\mathrm{V}$ type $\mathrm{H}^{+}$-ATPase is specific to vacuoles (Sze 1985; Nohzadeh Malakshah et al. 2007; Tanaka et al. 2004; Komatsu et al. 2007). As shown in Fig. 2, the $\mathrm{H}^{+}$-ATPase activity in the prepared plasma membrane samples from both differentiating xylem and phloem was sensitive to $\mathrm{Na}_{3} \mathrm{VO}_{4}$, but insensitive to $\mathrm{NaN}_{3}$ and $\mathrm{KNO}_{3}$. Quantitatively, $79.1 \%$ and $80.5 \%$ of the $\mathrm{H}^{+}$ATPase activity in the preparations of differentiating xylem and phloem cells were inhibited by $\mathrm{Na}_{3} \mathrm{VO}_{4}$, respectively. Meanwhile, only $9.3 \%$ and $10.6 \%$ of the $\mathrm{H}^{+}$-ATPase activity was inhibited by $\mathrm{NaN}_{3}$ and $11.7 \%$ and $12.9 \%$ by $\mathrm{KNO}_{3}$, in differentiating xylem and phloem cells, respectively. The sensitivity of the $\mathrm{H}^{+}$-ATPase activity to $\mathrm{Na}_{3} \mathrm{VO}_{4}$ but not to $\mathrm{NaN}_{3}$ or $\mathrm{KNO}_{3}$ suggests the predominance of $\mathrm{P}$-type $\mathrm{H}^{+}$-ATPase, which is specific to the plasma membrane. Overall the results demonstrated that the plasma membrane was isolated with a high degree of purity.

Protein isolation from the plasma membrane preparations

In previous studies, the plasma membrane proteins are generally directly analyzed using the SDS/acetone method after the aqueous two-phase separation (Santoni et al. 1998; Hurkman and Tanaka 1986; Nohzadeh Malakshah et al. 2007). However, when we first used this method to isolate the plasma membrane proteins from the poplar samples, the resolution quality of the subsequent electrophoresis analysis was poor (data not shown). Phenol extraction has been shown to enhance the qualitative and quantitative comparisons of plasma membrane proteins on 2-DE (Hurkman and Tanaka 1986; Saravanan and Rose 2004; Isaacson et al. 2006). To improve the resolution of the protein separation, the poplar plasma membrane preparation was first solublized by SDS buffer and then extracted with water-saturated phenol. After extraction, the plasma membrane proteins were divided into a soluble and insoluble portion. The soluble portion was analyzed on 2-DE, yielding a high 

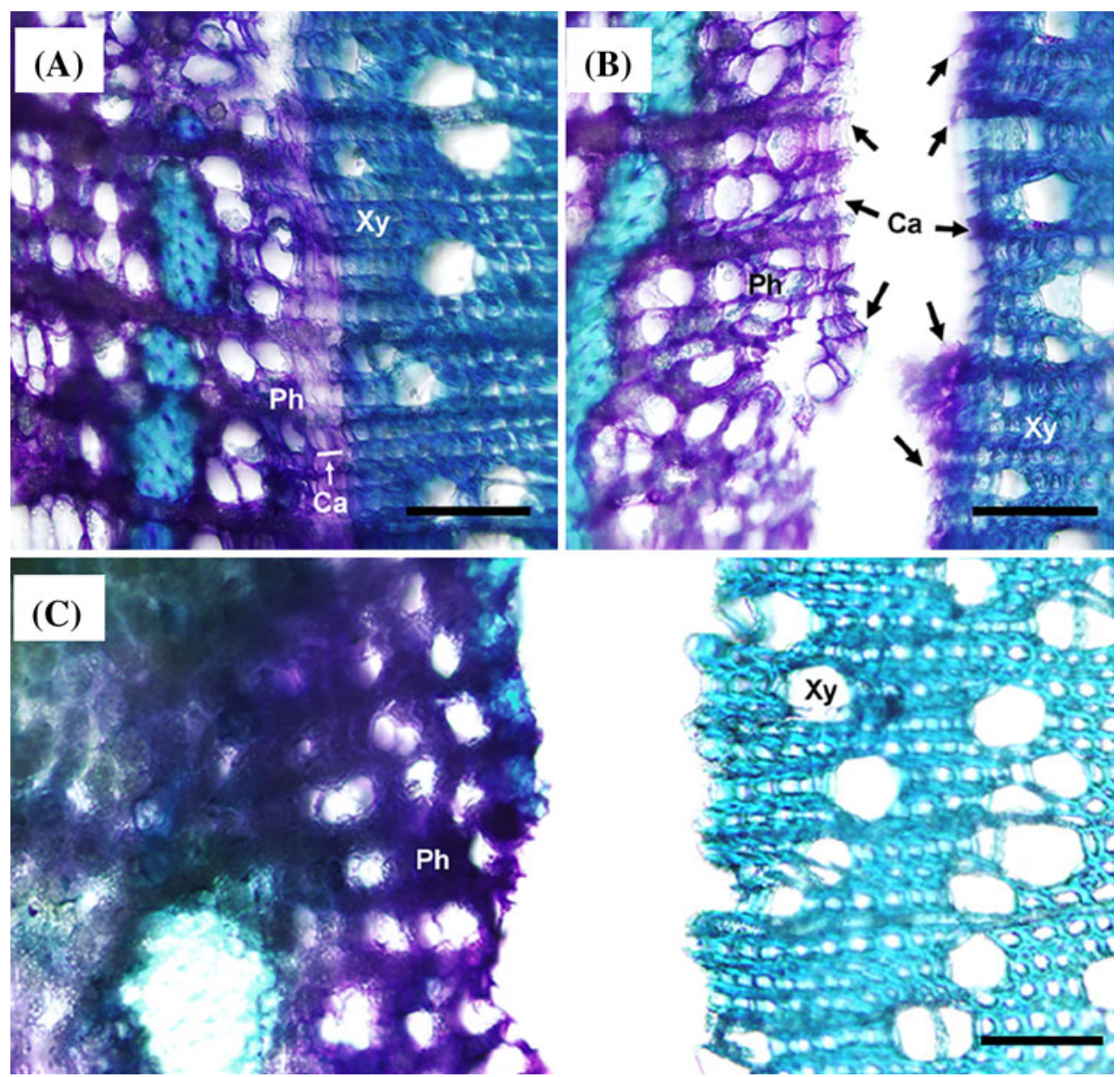

Fig. 1 Tissue sampling of Popolus secondary developing xylem and phloem. a Transverse section of Popolus stem before sampling. Four layers of cambium cells were underlined and indicated by arrows. b Transverse section of Popolus stem after bark separation. Cambium cells were found to be adhered to both xylem and phloem sides as

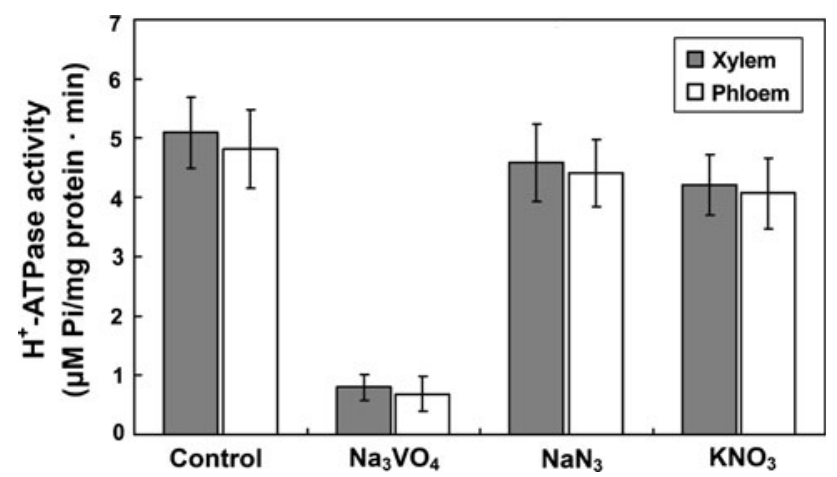

Fig. $2 \mathrm{H}^{+}$-ATPase activities in the isolated plasma membranes. Plasma membranes isolated from developing xylem (grey column) and phloem (white column) were measured for their $\mathrm{H}^{+}$-ATPase activities. $\mathrm{H}^{+}$-ATPase inhibitors, $\mathrm{Na}_{3} \mathrm{VO}_{4}, \mathrm{NaN}_{3}$ and $\mathrm{KNO}_{3}$ were used to examine the $\mathrm{H}^{+}$-ATPase type. $\mathrm{H}^{+}$-ATPase activity in the isolated plasma membranes was strongly inhibited by $\mathrm{Na}_{3} \mathrm{VO}_{4}$, but barely affected by $\mathrm{NaN}_{3}$ and $\mathrm{KNO}_{3}$

indicated by arrows. c Transverse section of Popolus stem after developing xylem and phloem tissues were harvested. The sections were stained with toluidine blue. Bar $50 \mu \mathrm{m}$. Xy xylem, Ca Cambium, $P h$ phloem

resolution separation, while the insoluble portion was partitioned on SDS-PAGE. Through this enhanced isolation procedure, plasma membrane proteins were effectively isolated and used for subsequent proteomic analysis.

Identification of the plasma membrane proteins by MS/MS analysis

The soluble portion of the plasma membrane proteins was profiled through $2 \mathrm{D}$ protein separation, which resulted in the identification of approximately 1,350 protein spots from differentiating xylem and about 1,351 protein spots from differentiating phloem (Fig. 3). The difference between xylem and phloem was compared using the Image Master Platinum software (v. 6.0). The results showed that while most of the protein spots matched each other in the two tissue samples, 55 protein spots were preferentially identified in 
Fig. 3 2-DE profile of plasma membrane proteins from developing xylem and phloem tissue in poplar. The soluble portion of the plasma membrane proteins was profiled though 2-DE. Arrow indicates proteins that were preferentially expressed in (a) xylem and (b) phloem tissue. The 1-D pI ranges are indicated at the top. $M W$ molecular weight

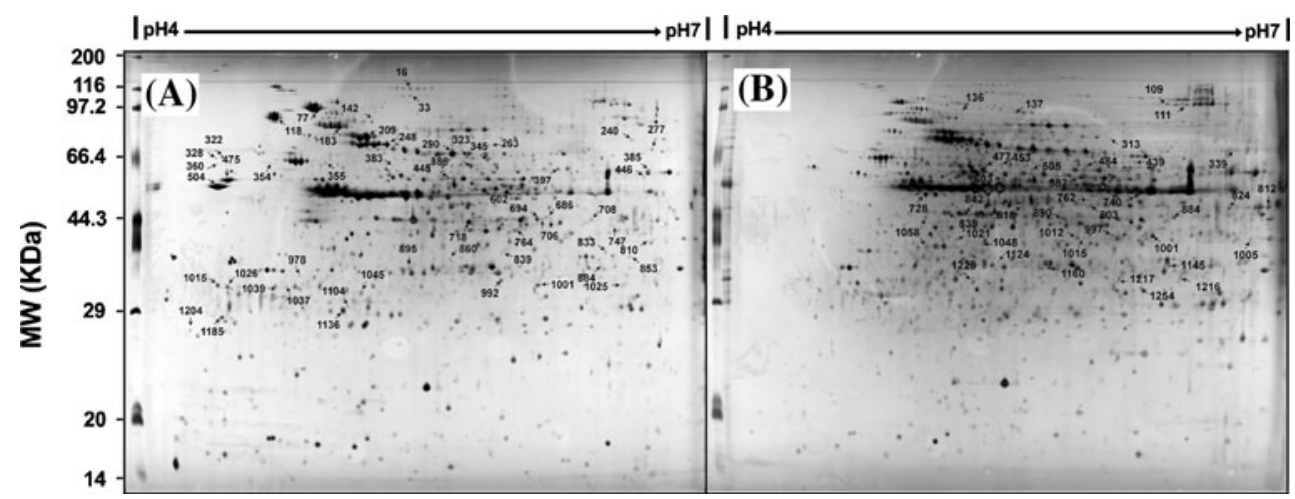

xylem and 40 in phloem. After MS/MS analysis and search against a Populus protein database (http://genome. jgi-psf.org/Poptr1_1/Poptr1_1.download.ftp.html; http://www. phytozome.net/poplar), the corresponding annotation and gene model of these proteins were characterized (Tables S1, S2).

Among the identified 95 proteins, their protein sequences were analyzed for the presence of a transmembrane domain using the TMHMM Server, v. 2.0 program (http:// www.cbs.dtu.dk/services/TMHMM/). Domain prediction indicated that only 8 proteins contained a single transmembrane domain and no protein contained multiple transmembrane domains. This suggests the majority of proteins in the soluble portion were not transmembrane and could therfore be peripheral or other proteins.

Meanwhile, the insoluble portion was partitioned by SDS-PAGE. After the gels were visualized, forty-six protein bands were detected and the proteins in each band were analyzed by LC-MS/MS analysis. The yielded peptide information was used to search a Populus protein database (http://genome.jgi-psf.org/Poptr1_1/Poptr1_1.down load.ftp.html; http://www.phytozome.net/poplar). Table 1 lists the proteins that were identified to contain at least one peptide unique to a particular protein. A total of 397 proteins were identified in xylem (Table 1 and Table S3) and 519 proteins identified in phloem (Table 1; Table S4).

A total of 678 different proteins were found in the phloem and xylem combined (Table 1; Tables S3, S4). Among them, xylem and phloem tissues shared 238 $(\sim 35 \%)$ proteins in common while $159(\sim 23 \%)$ proteins were only detected in xylem and $281(\sim 42 \%)$ proteins only in phloem (Fig. 4a). Among the detected proteins, 226 proteins $(\sim 33 \%)$ contained at least one transmembrane domain and the number of transmembrane domains varied in a range from 1 to 13 (Table 1; Fig. 4b). On the other hand, $452(\sim 67 \%)$ proteins were detected which did not contain transmembrane domains (Fig. 4b; Table S5). Within the transmembrane proteins, 133 of them $(\sim 59 \%)$ were identified in both xylem and phloem samples while 40 proteins were identified to be xylem-specific and 53 proteins were found to be phloem-specific (Table 1; Fig. 4c).
A comparison of the non-transmembrane proteins indicated that the two tissues had 105 proteins in common $(\sim 23 \%)$, while $119(\sim 26 \%)$ proteins were only identified in xylem and $228(\sim 51 \%)$ proteins were found only in phloem (Fig. 4d; Table S5).

In a previously reported study, analysis of the plasma membrane preparations from leaf, xylem and phloem/ cambium led to the identification of 213 out of 956 proteins as integral proteins(Nilsson et al. 2010). Here we identified 226 proteins with transmembrane domains from differentiating xylem and phloem which could be integral proteins. Although the total number of integral proteins detected in the two studies is similar, fewer non-transmembrane proteins (452) were detected in our study due to an additional separation procedure which resulted in the separation of more non-transmembrane proteins into a soluble portion.

The identification of 226 integral proteins in the specific tissues provides useful information to further investigate how plasma membrane proteins can regulate the differentiation of xylem and phloem tissues. For the non-transmembrane proteins it was difficult to determine whether they actually belonged to peripheral membrane proteins or another source.

Function classification of the plasma integral proteins from xylem and phloem

Being major functional players on the plasma membrane, integral proteins play important roles in signaling, cell inward/outward transportation and specific cell wall formation during cell differentiation. In the present study, the identified integral proteins were analyzed for their possible functions by sequence homology comparisons against the Arabidopsis Information Resource (TAIR) database. According to the annotation of their homolog genes in Arabidopsis and the results of structure domain analysis, a majority $(51 \%)$ of the integral proteins identified in the study had functions related to signaling (55 proteins), transportation (34 proteins), cell wall formation (27 proteins), or intracellular trafficking (17 proteins). The rest of 
Table 1 The transmembrane proteins identified from the plasma membrane of poplar differentiating xylem and phloem

\begin{tabular}{lcl}
\hline Protein ID Populus gene model & TMN NPDX NPDP $\begin{array}{l}\mathrm{Mw} \\
\text { (Da) }\end{array}$ & $\begin{array}{l}\text { Arabidopsis } \\
\text { Homolog }\end{array}$
\end{tabular} TAIR description

\begin{tabular}{|c|c|c|c|c|c|c|c|}
\hline \multicolumn{8}{|c|}{ Signal transduction (ST) } \\
\hline ST157 & gw1.XI.248.1 & 1 & 3 & 0 & 92433 & At4g21380 & Lectin-receptor-like protein kinase \\
\hline ST245 & eugene3.00191036 & 1 & 3 & 4 & 118701 & At4g03230 & Lectin-receptor-like protein kinase \\
\hline ST384 & gw1.XI.174.1 & 1 & 4 & 2 & 92581 & At4g21380 & Lectin-receptor-like protein kinase \\
\hline ST151 & eugene3.00131031 & 1 & 1 & 0 & 103786 & At3g56370 & LRR receptor-like protein kinase \\
\hline ST191 & gw1.29.518.1 & 1 & 3 & 5 & 72467 & At4g22130 & LRR receptor-like protein kinase \\
\hline ST247 & eugene3.00071196 & 1 & 2 & 3 & 121959 & At4g36180 & LRR receptor-like protein kinase \\
\hline ST330 & gw1.I.6094.1 & 1 & 5 & 4 & 112782 & At4g20940 & LRR receptor-like protein kinase \\
\hline ST358 & eugene3.00002215 & 1 & 12 & 13 & 103458 & At1g66150 & LRR receptor-like protein kinase \\
\hline ST359 & eugene 3.00040713 & 1 & 10 & 13 & 103230 & At1g66150 & LRR receptor-like protein kinase \\
\hline ST360 & fgenesh4_pg.C_LG_VI000556 & 1 & 23 & 33 & 100181 & At3g23750 & LRR receptor-like protein kinase \\
\hline ST363 & estExt_fgenesh4_pg.C_LG_XVIII1177 & 1 & 25 & 32 & 97108 & At3g23750 & LRR receptor-like protein kinase \\
\hline ST371 & eugene3.00160451 & 2 & 10 & 15 & 96327 & At5g06940 & LRR receptor-like protein kinase \\
\hline ST372 & eugene 3.01520008 & 2 & 12 & 20 & 95757 & At5g06940 & LRR receptor-like protein kinase \\
\hline ST374 & eugene3.00100968 & 1 & 12 & 13 & 100989 & At1g66150 & LRR receptor-like protein kinase \\
\hline ST375 & eugene3.00130345 & 1 & 4 & 4 & 100274 & At3g23750 & LRR receptor-like protein kinase \\
\hline ST386 & fgenesh4_pm.C_LG_XVIII000357 & 1 & 4 & 12 & 94301 & At2g24230 & LRR receptor-like protein kinase \\
\hline ST391 & fgenesh4_pg.C_LG_X001647 & 2 & 3 & 8 & 88905 & At3g51740 & LRR receptor-like protein kinase \\
\hline ST656 & eugene3.00190594 & 1 & 0 & 3 & 103453 & At3g56370 & LRR receptor-like protein kinase \\
\hline ST163* & eugene3.00060911 & 1 & 4 & 7 & 84818 & At3g51740 & LRR receptor-like protein kinase \\
\hline ST343* & fgenesh4_pm.C_LG_IV000169 & 1 & 12 & 13 & 108851 & At1g28440 & LRR receptor-like protein kinase \\
\hline ST373* & eugene3.00060471 & 1 & 4 & 10 & 96585 & At2g41820 & LRR receptor-like protein kinase \\
\hline ST385* & estExt_Genewise1_v1.C_LG_VII0023 & 1 & 6 & 6 & 94198 & At5g65700 & LRR receptor-like protein kinase \\
\hline ST390* & eugene3.00161196 & 2 & 7 & 7 & 89070 & At3g51740 & LRR receptor-like protein kinase \\
\hline ST263* & grail3.0010068301 & 1 & 3 & 2 & 123520 & At2g01950 & LRR receptor-like protein kinase, BRL2 \\
\hline ST8 & estExt_Genewise1_v1.C_290164 & 1 & 6 & 0 & 81436 & At1g14390 & Receptor-like protein kinase \\
\hline ST38 & fgenesh4_pm.C_LG_XIII000012 & 2 & 9 & 0 & 58921 & At1g56720 & Receptor-like protein kinase \\
\hline ST39 & fgenesh4_pg.C_scaffold_3857000001 & 1 & 6 & 0 & 20706 & At1g07650 & Receptor-like protein kinase \\
\hline ST173 & gw1.IV.3076.1 & 2 & 3 & 3 & 80902 & At4g04960 & Receptor-like protein kinase \\
\hline ST223 & eugene3.00100444 & 1 & 2 & 3 & 58001 & At1g67510 & Receptor-like protein kinase \\
\hline ST367 & eugene3.00160778 & 1 & 25 & 23 & 101857 & At2g37050 & Receptor-like protein kinase \\
\hline ST394 & grail3.0022032801 & 1 & 7 & 16 & 91591 & At3g46290 & Receptor-like protein kinase \\
\hline ST395 & grail3.0076008101 & 1 & 15 & 15 & 90959 & At3g51550 & Receptor-like protein kinase \\
\hline ST416 & fgenesh4_pg.C_LG_XVI000918 & 1 & 0 & 1 & 75825 & At3g55550 & Receptor-like protein kinase \\
\hline ST426 & gw1.XI.3269.1 & 1 & 0 & 6 & 71870 & At4g18640 & Receptor-like protein kinase \\
\hline ST165* & gw1.134.227.1 & 2 & 7 & 11 & 84850 & At5g54380 & Receptor-like protein kinase \\
\hline ST174* & gw1.XIII.3434.1 & 1 & 2 & 2 & 83247 & At4g03390 & Receptor-like protein kinase \\
\hline ST198* & eugene3.00131289 & 2 & 4 & 4 & 69403 & At5g58300 & Receptor-like protein kinase \\
\hline ST206* & estExt_fgenesh4_pg.C_LG_XV0398 & 2 & 2 & 4 & 67406 & At $1 \mathrm{~g} 48480$ & Receptor-like protein kinase \\
\hline ST207* & eugene3.00002256 & 1 & 3 & 5 & 67607 & At1g48480 & Receptor-like protein kinase \\
\hline ST208* & fgenesh4_pg.C_LG_IV000713 & 1 & 15 & 14 & 67735 & At1g48480 & Receptor-like protein kinase \\
\hline ST346* & gw1.XVI.567.1 & 1 & 11 & 24 & 107565 & At1g79620 & Receptor-like protein kinase \\
\hline ST351* & gw1.28.1090.1 & 1 & 7 & 10 & 104012 & At1g79620 & Receptor-like protein kinase \\
\hline ST366* & eugene3.00060962 & 2 & 19 & 20 & 97278 & At3g51550 & Receptor-like protein kinase \\
\hline ST387* & gw1.I.6134.1 & 2 & 5 & 9 & 93233 & At1g30570 & Receptor-like protein kinase \\
\hline ST388* & eugene3.00110972 & 2 & 7 & 10 & 92737 & At5g54380 & Receptor-like protein kinase \\
\hline ST392* & grail3.0001120501 & 2 & 10 & 13 & 90916 & At3g46290 & Receptor-like protein kinase \\
\hline
\end{tabular}


Table 1 continued

\begin{tabular}{|c|c|c|c|c|c|c|c|}
\hline Protein ID & Populus gene model & TMN & NPDX & NPDP & $\begin{array}{l}\mathrm{Mw} \\
(\mathrm{Da})\end{array}$ & $\begin{array}{l}\text { Arabidopsis } \\
\text { Homolog }\end{array}$ & TAIR description \\
\hline ST393* & gw1.I.1449.1 & 1 & 8 & 12 & 91099 & At3g46290 & Receptor-like protein kinase \\
\hline ST665* & gw1.86.291.1 & 1 & 0 & 5 & 101997 & At5g49760 & Receptor-like protein kinase \\
\hline ST651 & gw1.VIII.2924.1 & 2 & 0 & 3 & 111360 & At2g01830 & Receptor histidine kinase, CRE1 \\
\hline ST221 & fgenesh4_pg.C_scaffold_21924000001 & 1 & 8 & 6 & 19070 & No hit & Hybrid histidine kinase \\
\hline ST7 & estExt_Genewise1_v1.C_LG_XVIII0587 & 1 & 2 & 0 & 84654 & At3g43220 & Phosphoinositide phosphatase \\
\hline ST75 & eugene3.00150591 & 2 & 9 & 0 & 39135 & At5g63050 & Emb2759, embryo defective 2759 \\
\hline ST179 & gw1.41.218.1 & 1 & 9 & 8 & 79168 & At1g34550 & Emb2756, embryo defective 2756 \\
\hline ST284* & gw1.II.2836.1 & 5 & 6 & 5 & 43018 & At3g25290 & Auxin-responsive protein \\
\hline ST356 & fgenesh4_pm.C_LG_IX000015 & 3 & 2 & 2 & 99384 & At4g35290 & Glutamate receptor \\
\hline \multicolumn{8}{|c|}{ Transporter (TR) } \\
\hline TR531 & gw1.I.4875.1 & 6 & 0 & 4 & 11815 & At3g13220 & $\mathrm{ABC}$ transporter \\
\hline TR578* & estExt_Genewise1_v1.C_LG_II3719 & 10 & 0 & 19 & 132305 & At2g47000 & $\mathrm{ABC}$ transporter \\
\hline TR5 & gw1.44.184.1 & 10 & 11 & 0 & 84023 & At3g21250 & $\mathrm{ABC}$ transporter \\
\hline TR119 & eugene3.00061718 & 7 & 1 & 0 & 26131 & At2g25810 & Aquaporin \\
\hline TR271 & estExt_Genewise1_v1.C_LG_XVI2799 & 6 & 2 & 2 & 30400 & At2g 37170 & Aquaporin \\
\hline TR283 & estExt_fgenesh4_pg.C_LG_IX1411 & 6 & 2 & 3 & 30433 & At2g37170 & Aquaporin \\
\hline TR565 & eugene 3.00011331 & 7 & 0 & 1 & 24955 & At3g 16240 & Aquaporin \\
\hline TR256* & eugene3.00280238 & 6 & 3 & 3 & 26043 & At2g36830 & Aquaporin \\
\hline TR281* & grail3.0045020302 & 6 & 2 & 2 & 29585 & At4g35100 & Aquaporin \\
\hline TR282* & estExt_Genewise1_v1.C_LG_III0271 & 6 & 4 & 5 & 30753 & At4g00430 & Aquaporin \\
\hline TR296* & grail3.0049030302 & 6 & 3 & 3 & 30949 & At4g00430 & Aquaporin \\
\hline TR303* & eugene 3.00102165 & 6 & 8 & 9 & 30303 & At3g54820 & Aquaporin \\
\hline TR543* & estExt_fgenesh4_pm.C_LG_XVI0408 & 6 & 0 & 1 & 30621 & At4g00430 & Aquaporin \\
\hline TR389 & gw1.VI.1514.1 & 6 & 1 & 1 & 89766 & At4g30110 & Cadmium-transporting ATPase \\
\hline TR211* & estExt_Genewise1_v1.C_LG_I4955 & 11 & 2 & 2 & 64816 & At1g53210 & Calcium-binding EF hand protein \\
\hline TR143 & gw1.148.178.1 & 9 & 11 & 0 & 106054 & At1g07670 & Calcium-transporting ATPase \\
\hline TR314 & fgenesh4_pg.C_LG_IX001309 & 8 & 16 & 8 & 116598 & At1g07670 & Calcium-transporting ATPase \\
\hline TR600 & gw1.135.25.1 & 8 & 0 & 12 & 124965 & At5g23630 & Cation-transporting ATPase \\
\hline TR145 & fgenesh4_pg.C_LG_III000552 & 8 & 3 & 0 & 107764 & At5g44790 & Copper-transporting ATPase \\
\hline TR357 & eugene 3.00010321 & 8 & 6 & 7 & 105221 & At1g63440 & Copper-transporting ATPase, HMA5 \\
\hline TR299* & estExt_fgenesh4_pg.C_LG_II0267 & 10 & 2 & 2 & 41736 & At1g75500 & MtN21-like protein \\
\hline TR300* & estExt_fgenesh4_pg.C_LG_V1470 & 10 & 4 & 5 & 41898 & At1g75500 & MtN21-like protein \\
\hline TR33 & gw1.158.66.1 & 8 & 2 & 0 & 61016 & At1g72480 & Multiple transmembrane protein \\
\hline TR336 & eugene3.00011076 & 5 & 8 & 10 & 106164 & At1g52780 & Multiple transmembrane protein \\
\hline TR225 & gw1.XVI.376.1 & 8 & 1 & 1 & 57747 & At1g61670 & Multiple transmembrane protein \\
\hline TR265 & gw1.XV.1560.1 & 7 & 1 & 1 & 45776 & At5g33320 & Phosphate translocator \\
\hline TR495 & fgenesh4_pg.C_LG_I000711 & 8 & 0 & 15 & 142941 & At1g17500 & Phospholipid-transporting ATPase \\
\hline TR349* & gw1.XII.988.1 & 8 & 24 & 19 & 104609 & At5g62670 & Plasma membrane $\mathrm{H}^{+}$-ATPase \\
\hline TR361* & gw1.XV.1202.1 & 8 & 18 & 15 & 104511 & At5g62670 & Plasma membrane $\mathrm{H}^{+}$-ATPase \\
\hline TR182* & gw1.66.623.1 & 11 & 2 & 2 & 79166 & At4g35300 & Sugar transporter \\
\hline TR60* & fgenesh4_pg.C_LG_II002606 & 12 & 1 & 0 & 52470 & At1g75220 & Sugar transporter \\
\hline TR379* & estExt_fgenesh4_pg.C_LG_IX0438 & 6 & 14 & 12 & 93123 & At2g21410 & VHA-A2 \\
\hline TR158 & fgenesh4_pg.C_LG_II000263 & 6 & 2 & 0 & 91988 & At2g21410 & VHA-A2 \\
\hline TR380* & estExt_fgenesh4_pm.C_LG_IV0476 & 6 & 12 & 10 & 92736 & At4g39080 & VHA-A2 \\
\hline \multicolumn{8}{|c|}{ Cell wall formation and carbohydrate metabolism (CW) } \\
\hline CW662 & estExt_fgenesh4_pg.C_LG_X0451 & 1 & 0 & 2 & 96660 & At1g67490 & Alpha-glucosidase I \\
\hline CW258 & eugene3.00011928 & 1 & 3 & 5 & 120061 & At5g14950 & Alpha-mannosidase \\
\hline
\end{tabular}


Table 1 continued

\begin{tabular}{|c|c|c|c|c|c|c|c|}
\hline Protein ID & Populus gene model & TMN & NPDX & NPDP & $\begin{array}{l}\mathrm{Mw} \\
(\mathrm{Da})\end{array}$ & $\begin{array}{l}\text { Arabidopsis } \\
\text { Homolog }\end{array}$ & TAIR description \\
\hline CW304 & estExt_fgenesh4_pm.C_LG_III0447 & 1 & 5 & 7 & 38374 & At5g53340 & Beta-1,3-galactosyltransferase \\
\hline CW232* & estExt_fgenesh4_pg.C_13980001 & 8 & 5 & 6 & 121143 & At4g32410 & CesA1-A \\
\hline CW270 & eugene3.00060479 & 8 & 3 & 4 & 118944 & At5g05170 & CesA3-A \\
\hline CW234 & estExt_fgenesh4_pg.C_LG_IX0979 & 8 & 4 & 2 & 120652 & At5g05170 & CesA3-C \\
\hline CW302 & eugene3.00160483 & 8 & 7 & 13 & 119929 & At5g05170 & CesA3-D \\
\hline CW308* & eugene3.00002636 & 8 & 10 & 12 & 118579 & At5g44030 & CesA4 \\
\hline CW278 & fgenesh4_pm.C_LG_XIII000084 & 8 & 14 & 8 & 122381 & At5g64740 & CesA6-E \\
\hline CW310* & gw1.XVIII.3152.1 & 8 & 10 & 22 & 116943 & At5g17420 & CesA7-A \\
\hline CW289* & estExt_Genewise1_v1.C_LG_VI2188 & 8 & 12 & 25 & 116336 & At5g17420 & CesA7-B \\
\hline CW341* & gw1.XI.3218.1 & 8 & 14 & 16 & 110349 & At4g18780 & CesA8-A \\
\hline CW321 & eugene3.00040363 & 8 & 19 & 18 & 114553 & At4g18780 & CesA8-B \\
\hline CW18 & estExt_fgenesh4_pg.C_LG_X0013 & 1 & 1 & 0 & 70938 & At4g16120 & Cobl (Cobra-like protein) \\
\hline CW229* & estExt_fgenesh4_pm.C_LG_V0631 & 1 & 2 & 5 & 57112 & At1g19940 & Endo-beta-1,4-glucanase family protein \\
\hline CW196* & grail3.0263001401 & 1 & 5 & 9 & 68495 & At5g49720 & $\begin{array}{l}\text { Endo-beta-1,4-glucanase, KOR } \\
\text { homolog }\end{array}$ \\
\hline CW220 & eugene3.00071182 & 1 & 13 & 17 & 58428 & At4g36220 & Ferulate-5-hydroxylase \\
\hline CW216 & estExt_fgenesh4_pg.C_LG_III0527 & 1 & 13 & 11 & 59876 & At1g17270 & $\begin{array}{l}\text { GDP-fucose protein-o- } \\
\text { fucosyltransferase }\end{array}$ \\
\hline CW644 & gw1.II.3117.1 & 1 & 0 & 2 & 113954 & At4g01210 & Glycosyltransferase family protein \\
\hline CW253 & gw1.VII.2855.1 & 2 & 5 & 7 & 48551 & At4g36890 & GT family 43 protein, IRX14 \\
\hline CW231 & estExt_Genewise1_v1.C_LG_V4069 & 1 & 5 & 7 & 56558 & At5g67230 & GT family 43 protein, IRX14 homolog \\
\hline CW461 & eugene3.00130489 & 5 & 0 & 2 & 59094 & At5g18480 & GT family 8 protein \\
\hline CW114 & eugene3.00190332 & 8 & 2 & 0 & 128266 & At3g03050 & PtCslD6 \\
\hline CW65 & eugene3.00880019 & 1 & 7 & 0 & 50504 & At3g23820 & UDP-glucuronate 4-epimerase \\
\hline CW250* & estExt_Genewise1_v1.C_LG_XVI2527 & 1 & 12 & 9 & 48929 & At3g62830 & UDP-glucuronic acid decarboxylase \\
\hline CW246* & eugene3.00140737 & 1 & 9 & 9 & 49744 & At3g62830 & UDP-glucuronic acid decarboxylase \\
\hline CW520* & fgenesh4_pm.C_LG_II000873 & 1 & 0 & 1 & 48435 & At2g47650 & UDP-glucuronic acid decarboxylase \\
\hline \multicolumn{8}{|c|}{ Intracellular trafficking (IT) } \\
\hline IT144* & gw1.I.9637.1 & 1 & 1 & 0 & 107845 & At1g52360 & Coatomer beta subunit \\
\hline IT261 & grail3.0020010802 & 1 & 20 & 18 & 33638 & At2g19950 & Golgin-84 \\
\hline IT181 & estExt_Genewise1_v1.C_440243 & 1 & 2 & 4 & 79746 & At5g45160 & GTP-binding protein \\
\hline IT396 & gw1.XV.2621.1 & 1 & 2 & 2 & 90530 & At5g45160 & GTP-binding protein \\
\hline IT410 & gw1.246.2.1 & 1 & 0 & 11 & 79912 & At5g45160 & GTP-binding protein \\
\hline IT592* & Eugene3.00060912 & 3 & 0 & 3 & 23984 & At2g38360 & Prenylated rab acceptor \\
\hline IT601 & Fgenesh4_pm.C_LG_XIX000312 & 4 & 0 & 1 & 22640 & At2g38360 & Prenylated rab acceptor \\
\hline IT17 & estExt_fgenesh4_pm.C_700083 & 1 & 5 & 0 & 71860 & At5g27540 & Rac-GTP binding protein \\
\hline IT288 & Eugene3.00140364 & 3 & 2 & 2 & 28100 & At2g46170 & Reticulon family protein \\
\hline IT574 & gw1.122.33.1 & 3 & 0 & 8 & 29014 & At4g23630 & Reticulon family protein \\
\hline IT617* & estExt_fgenesh4_pm.C_LG_X0012 & 1 & 0 & 7 & 28624 & At3g17440 & SNARE, protein transporter \\
\hline IT291 & gw1.X.834.1 & 1 & 12 & 18 & 41243 & At2g03510 & Synaptobrevin-related protein \\
\hline IT106* & estExt_fgenesh4_pg.C_LG_XV0909 & 1 & 2 & 0 & 24596 & At1g04760 & Synaptobrevin-related protein \\
\hline IT315 & estExt_fgenesh4_pm.C_LG_XIX0109 & 1 & 7 & 9 & 34275 & At3g03800 & Syntaxin 131 \\
\hline IT628 & estExt_fgenesh4_pg.C_290237 & 1 & 0 & 5 & 30179 & At4g17730 & Syntaxin 23 \\
\hline IT629* & Eugene3.00280153 & 1 & 0 & 15 & 30759 & At3g09740 & Syntaxin 71 \\
\hline IT631* & estExt_fgenesh4_pg.C_LG_XVI0751 & 1 & 0 & 15 & 29693 & At3g09740 & Syntaxin 71 \\
\hline \multicolumn{8}{|c|}{ Function unknown (FU) } \\
\hline FU161 & estExt_Genewise1_v1.C_LG_XVI1567 & 3 & 2 & 2 & 85234 & At3g57880 & $\mathrm{C}_{2}$ domain-containing protein \\
\hline
\end{tabular}


Table 1 continued

\begin{tabular}{|c|c|c|c|c|c|c|c|}
\hline Protein ID & Populus gene model & $\mathrm{TMN}$ & NPDX & NPDP & $\begin{array}{l}\mathrm{Mw} \\
(\mathrm{Da})\end{array}$ & $\begin{array}{l}\text { Arabidopsis } \\
\text { Homolog }\end{array}$ & TAIR description \\
\hline FU498 & gw1.VIII.297.1 & 3 & 0 & 2 & 51259 & At4g14240 & CBS domain-containing protein \\
\hline FU50* & gw1.X.4147.1 & 3 & 3 & 0 & 54367 & At4g14240 & CBS domain-containing protein \\
\hline FU215 & fgenesh4_pg.C_scaffold_70000199 & 1 & 6 & 7 & 18462 & No hit & Duf1068 \\
\hline FU559 & gw1.X.1601.1 & 1 & 0 & 2 & 43883 & At2g40320 & Duf213 \\
\hline FU239 & estExt_fgenesh4_pg.C_LG_X1673 & 1 & 6 & 4 & 53094 & At3g55990 & Duf231 \\
\hline FU448 & estExt_fgenesh4_pg.C_LG_XVI0458 & 1 & 0 & 2 & 64313 & At5g06700 & Duf231 \\
\hline FU517 & estExt_Genewise1_v1.C_LG_XI3238 & 1 & 0 & 11 & 47601 & At1g29200 & Duf246 \\
\hline FU170 & estExt_fgenesh4_pg.C_LG_II2588 & 1 & 1 & 2 & 83319 & At1g19430 & Duf248 \\
\hline FU184 & eugene 3.00080530 & 1 & 5 & 7 & 75654 & At2g39750 & Duf248 \\
\hline FU193 & estExt_fgenesh4_pg.C_290162 & 1 & 13 & 15 & 68792 & At4g18030 & Duf248 \\
\hline FU194 & estExt_fgenesh4_pg.C_290313 & 1 & 1 & 3 & 68364 & At1g31850 & Duf248 \\
\hline FU429 & gw1.I.2672.1 & 1 & 0 & 2 & 69704 & At5g14430 & Duf248 \\
\hline FU434 & estExt_fgenesh4_pg.C_LG_VIII1310 & 1 & 0 & 5 & 69331 & At1g26850 & Duf248 \\
\hline FU435 & estExt_fgenesh4_pg.C_LG_X0857 & 1 & 0 & 4 & 69638 & At1g26850 & Duf248 \\
\hline FU442 & fgenesh4_pg.C_LG_XIV000203 & 1 & 0 & 2 & 67005 & At4g00740 & Duf248 \\
\hline FU160* & fgenesh4_pg.C_LG_V000057 & 1 & 5 & 7 & 87575 & At5g64030 & Duf248 \\
\hline FU195* & estExt_fgenesh4_pg.C_LG_V1395 & 1 & 4 & 11 & 69308 & At1g04430 & Duf248 \\
\hline FU381* & estExt_Genewise1_v1.C_LG_VII1503 & 1 & 7 & 8 & 92340 & At5g64030 & Duf248 \\
\hline FU162 & estExt_fgenesh4_pg.C_LG_VI0421 & 1 & 2 & 6 & 87693 & At2g41770 & Duf288 \\
\hline FU47 & eugene 3.00080638 & 1 & 5 & 0 & 55079 & At3g55990 & Duf321 \\
\hline FU79 & eugene3.00050506 & 1 & 1 & 0 & 35496 & At5g67210 & Duf579 \\
\hline FU85 & gw1.XIX.1870.1 & 1 & 1 & 0 & 33668 & At1g33800 & Duf579 \\
\hline FU103 & gw1.41.566.1 & 1 & 2 & 0 & 30634 & At1g33800 & Duf579 \\
\hline FU120 & gw1.86.114.1 & 1 & 2 & 0 & 32443 & At1g09610 & Duf579 \\
\hline FU297 & gw1.VII.2881.1 & 1 & 5 & 6 & 36120 & At5g67210 & Duf579 \\
\hline FU449 & eugene3.01240052 & 1 & 0 & 1 & 66720 & At1g28240 & Duf616 \\
\hline FU273 & estExt_fgenesh4_pm.C_LG_IX0699 & 1 & 1 & 1 & 43885 & At $2 \mathrm{~g} 28310$ & Duf707 \\
\hline FU185 & eugene 3.00570132 & 1 & 2 & 3 & 77002 & At3g51050 & Fg-gap repeat-containing protein \\
\hline FU605 & eugene3.01200101 & 1 & 0 & 16 & 131690 & No hit & $\begin{array}{l}\text { Tir-nbs-tir type disease resistance } \\
\text { protein }\end{array}$ \\
\hline FU430 & fgenesh4_pg.C_LG_IX000173 & 1 & 0 & 12 & 64478 & At5g21990 & Trp-containing protein \\
\hline FU340 & fgenesh4_pm.C_LG_VI000737 & 2 & 4 & 5 & 109593 & At5g11560 & Unknown protein \\
\hline FU9 & eugene3.00400141 & 2 & 1 & 0 & 83569 & At3g60380 & Unknown protein \\
\hline FU13 & eugene3.00081921 & 1 & 3 & 0 & 75211 & At3g06150 & Unknown protein \\
\hline FU30 & estExt_Genewise1_v1.C_LG_IV3066 & 1 & 2 & 0 & 66269 & At1g28240 & Unknown protein \\
\hline FU66 & fgenesh4_pm.C_LG_I000553 & 1 & 1 & 0 & 50584 & At3g 16200 & Unknown protein \\
\hline FU76 & eugene3.00130055 & 3 & 1 & 0 & 20866 & At1g09330 & Unknown protein \\
\hline FU90 & eugene3.00180117 & 1 & 1 & 0 & 44673 & At5g11730 & Unknown protein \\
\hline FU166 & eugene3.00180314 & 1 & 5 & 6 & 85916 & At5g11560 & Unknown protein \\
\hline FU167 & eugene3.01340010 & 1 & 1 & 1 & 87295 & At4g27290 & Unknown protein \\
\hline FU187 & gw1.XIX.2110.1 & 1 & 6 & 5 & 79107 & At1g34550 & Unknown protein \\
\hline FU214 & eugene 3.00141171 & 1 & 2 & 3 & 65644 & At2g04280 & Unknown protein \\
\hline FU252 & estExt_fgenesh4_pm.C_LG_X0570 & 1 & 2 & 3 & 48530 & At4g16170 & Unknown protein \\
\hline FU267 & eugene 3.00160295 & 1 & 3 & 3 & 36620 & At3g56750 & Unknown protein \\
\hline FU269 & estExt_Genewise1_v1.C_1400180 & 4 & 2 & 2 & 23818 & At5g56020 & Unknown protein \\
\hline FU293 & estExt_fgenesh4_pg.C_LG_IX0791 & 1 & 1 & 1 & 28677 & At3g49720 & Unknown protein \\
\hline FU335 & eugene3.01240046 & 3 & 2 & 6 & 107178 & At4g21700 & Unknown protein \\
\hline
\end{tabular}


Table 1 continued

\begin{tabular}{|c|c|c|c|c|c|c|c|}
\hline Protein ID & Populus gene model & $\mathrm{TMN}$ & NPDX & NPDP & $\begin{array}{l}\mathrm{Mw} \\
(\mathrm{Da})\end{array}$ & $\begin{array}{l}\text { Arabidopsis } \\
\text { Homolog }\end{array}$ & TAIR description \\
\hline FU397 & gw1.I.2846.1 & 1 & 4 & 3 & 90914 & At3g01720 & Unknown protein \\
\hline FU419 & eugene3.00081920 & 1 & 0 & 3 & 75132 & At3g06150 & Unknown protein \\
\hline FU476 & fgenesh4_pg.C_scaffold_192000004 & 1 & 0 & 8 & 54884 & No hit & Unknown protein \\
\hline FU481 & gw1.28.115.1 & 1 & 0 & 11 & 53253 & At5g20680 & Unknown protein \\
\hline FU513 & estExt_Genewise1_v1.C_LG_X3098 & 2 & 0 & 1 & 50210 & At1g16860 & Unknown protein \\
\hline FU537 & estExt_Genewise1_v1.C_LG_XVIII0915 & 1 & 0 & 5 & 30200 & At5g11890 & Unknown protein \\
\hline FU597 & eugene 3.00060357 & 1 & 0 & 2 & 28946 & At3g56750 & Unknown protein \\
\hline FU669 & fgenesh4_pg.C_LG_XVIII001074 & 1 & 0 & 5 & 15019 & No hit & Unknown protein \\
\hline FU292* & estExt_fgenesh4_pm.C_LG_II1168 & 4 & 2 & 3 & 31667 & At2g20230 & Unknown protein \\
\hline FU62* & eugene3.00080044 & 2 & 2 & 0 & 51817 & At1g16860 & Unknown protein \\
\hline FU307 & grail3.0010063802 & 5 & 1 & 1 & 39805 & At1g68070 & Zinc finger family protein \\
\hline FU569 & eugene3.00141007 & 2 & 0 & 7 & 42604 & At5g41060 & Zinc finger family protein \\
\hline \multicolumn{8}{|c|}{ Unclassified (UC) } \\
\hline UC333* & eugene3.00170157 & 1 & 4 & 7 & 109369 & At2g32730 & $26 \mathrm{~s}$ proteasome regulatory subunit \\
\hline UC459 & estExt_fgenesh4_pg.C_LG_II2102 & 10 & 0 & 2 & 59079 & At1g05820 & Aspartic-type endopeptidase \\
\hline $\mathrm{UC} 12$ & fgenesh4_pg.C_scaffold_28000065 & 7 & 15 & 0 & 197526 & At2g03140 & CAAX amino terminal protease \\
\hline UC92 & gw1.XVI.2464.1 & 1 & 22 & 0 & 116765 & At2g36200 & Kinesin motor protein-related \\
\hline UC240* & eugene 3.00102346 & 2 & 2 & 2 & 51843 & At1g16860 & Merozoite surface protein-related \\
\hline UC464 & grail3.0003069401 & 1 & 0 & 14 & 56153 & At1g77510 & Protein disulfide isomerase \\
\hline UC199 & estExt_fgenesh4_pm.C_LG_II0175 & 1 & 6 & 9 & 69918 & At1g04430 & SAM-dependent methyltransferase \\
\hline UC243 & fgenesh4_pg.C_LG_I000592 & 1 & 10 & 11 & 55513 & At1g11680 & Sterol 14-demethylase \\
\hline UC557 & estExt_fgenesh4_pg.C_LG_II0150 & 1 & 0 & 8 & 40593 & At1g20330 & Sterol C-24 methyltransferase \\
\hline UC213 & fgenesh4_pg.C_LG_VIII000736 & 1 & 16 & 22 & 65583 & At3g19820 & Sterol C-24 reductase \\
\hline UC280* & estExt_Genewise1_v1.C_290374 & 4 & 1 & 1 & 31173 & At1g32400 & TOM2A \\
\hline \multicolumn{8}{|c|}{ Possible contaminants (PC) } \\
\hline PC210* & estExt_fgenesh4_pg.C_LG_X1518 & 1 & 16 & 16 & 65824 & At3g19820 & 24-dehydrocholesterol reductase \\
\hline PC620 & gw1.2627.7.1 & 1 & 0 & 7 & 21843 & No hit & $\begin{array}{l}\text { 3-octaprenyl-4-hydroxybenzoate } \\
\text { carboxy-lyase }\end{array}$ \\
\hline PC108* & gw1.64.623.1 & 3 & 17 & 0 & 40899 & At4g28390 & ADP/ATP antiporter \\
\hline PC279* & estExt_fgenesh4_pg.C_LG_I1918 & 3 & 23 & 24 & 42074 & At5g 13490 & ADP/ATP antiporter \\
\hline PC295* & gw1.IX.3274.1 & 3 & 4 & 5 & 40779 & At5g13490 & ADP/ATP antiporter \\
\hline PC242 & fgenesh4_pm.C_LG_XIX000083 & 1 & 1 & 1 & 51119 & At5g 18280 & Apyrase \\
\hline $\mathrm{PC} 248 *$ & estExt_fgenesh4_pg.C_LG_VII1013 & 1 & 5 & 5 & 48905 & At5g66680 & $\begin{array}{l}\text { Dolichyl-diphosphooligosaccharide- } \\
\text { protein glycotransferase }\end{array}$ \\
\hline PC51 & gw1.III.1232.1 & 9 & 3 & 0 & 54282 & At1g10950 & Endomembrane protein 70 , putative \\
\hline PC202 & grail3.0038018602 & 10 & 15 & 4 & 67928 & At2g01970 & Endomembrane protein 70 , putative \\
\hline PC443 & eugene 3.00440214 & 10 & 0 & 3 & 68212 & At5g 37310 & Endomembrane protein 70 , putative \\
\hline PC583 & estExt_Genewise1_v1.C_27420001 & 10 & 0 & 3 & 41321 & At5g37310 & Endomembrane protein 70 , putative \\
\hline PC188* & eugene3.00061953 & 10 & 3 & 2 & 73326 & At5g 10840 & Endomembrane protein 70 , putative \\
\hline $\mathrm{PC} 424 *$ & estExt_fgenesh4_pm.C_LG_I0291 & 1 & 0 & 3 & 73510 & At5g42020 & ER luminal-binding protein \\
\hline PC501 & fgenesh4_pg.C_scaffold_896000002 & 1 & 0 & 10 & 50806 & No hit & Integrase protein \\
\hline PC275 & estExt_fgenesh4_pm.C_LG_XV0031 & 1 & 8 & 5 & 43274 & At4g27680 & Msp1 protein, putative \\
\hline PC46 & eugene3.00031308 & 1 & 11 & 0 & 55427 & At1g11680 & $\begin{array}{l}\text { Probable obtusifoliol 1,4-alpha- } \\
\text { demethylase }\end{array}$ \\
\hline PC40 & fgenesh4_pg.C_LG_II001639 & 1 & 4 & 0 & 58769 & At1g01120 & Putative beta-ketoacyl-CoA synthase \\
\hline PC180 & gw1.XVIII.267.1 & 1 & 9 & 5 & 80153 & At1g15690 & Vacuolar $\mathrm{H}^{+}$-pyrophosphatase \\
\hline PC183* & estExt_fgenesh4_pg.C_1520062 & 13 & 7 & 4 & 80345 & At1g15690 & Vacuolar $\mathrm{H}^{+}$-pyrophosphatase \\
\hline
\end{tabular}


Table 1 continued

\begin{tabular}{llcccccl}
\hline Protein ID & Populus gene model & TMN & NPDX & NPDP & $\begin{array}{l}\text { Mw } \\
\text { (Da) }\end{array}$ & $\begin{array}{l}\text { Arabidopsis } \\
\text { Homolog }\end{array}$ & TAIR description \\
\hline PC432 & fgenesh4_pm.C_LG_I000245 & 2 & 0 & 2 & 71461 & At1g30900 & Vacuolar sorting receptor \\
PC433 & fgenesh4_pg.C_LG_VI000894 & 2 & 0 & 2 & 69479 & At3g52850 & Vacuolar sorting receptor \\
PC549 & estExt_fgenesh4_pm.C_LG_III0520 & 1 & 0 & 6 & 12202 & At1g30900 & Vacuolar sorting receptor \\
PC23 & estExt_Genewise1_v1.C_LG_I4100 & 2 & 5 & 0 & 69567 & At2g14740 & Vacuolar-sorting receptor \\
\hline
\end{tabular}

TMN: transmembrane domain number, NPDX: number of peptides detected in xylem, NPDP: number of peptides detected in phloem. Note: * indicates that the protein was also detected in Nilsson's study

Fig. 4 Distribution of the identified plasma membrane proteins from developing xylem and phloem tissue. (a) Total number of the identified plasma membrane proteins in the insoluble portion, (b) proportion of the transmembrane and nontransmembrane proteins,

(c) distribution of transmembrane proteins and (d) distribution of nontransmembrane proteins
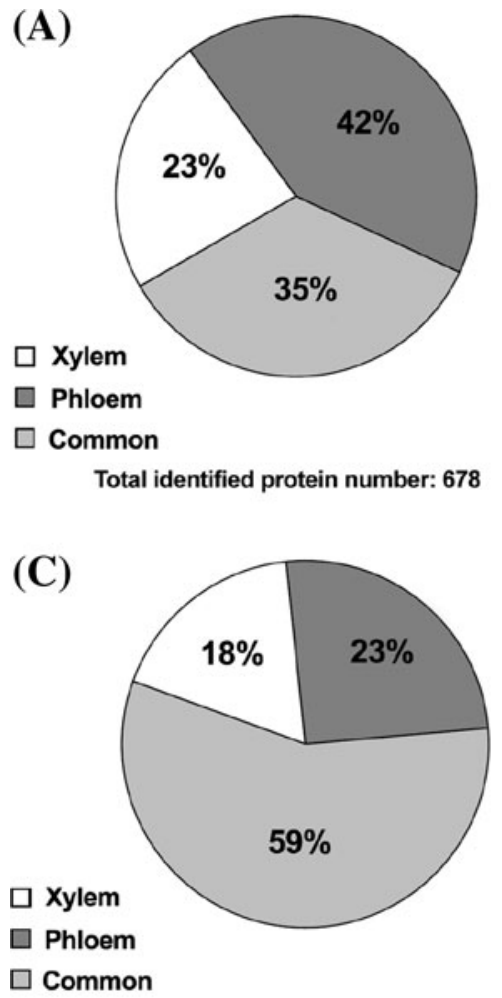

Identified transmembrane protein number: 226
(B)

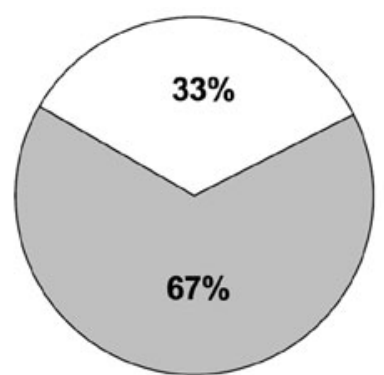

$\square$ Transmembrane

$\square$ Non-transmembrane

Total identified protein number: 678

(D)

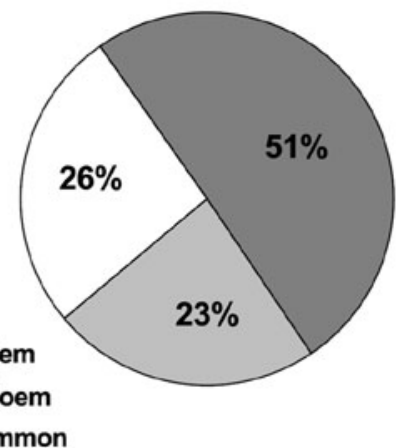

Identified non-transmembrane protein number: 452 the proteins could not be classified (11 proteins) due to either unknown function (59 proteins) or possible containments (23 proteins) (Table 1; Fig. 5a, b). Many overlapping proteins were identified in xylem and phloem tissues (Fig. 5b) which may reflect a set of similar biological processes such as intensive cell wall biosynthesis that occur over the course of the differentiation of both sets of tissues. At the same time, a number of proteins were also identified as being specifically related to either xylem or phloem formation, suggesting that these proteins may be involved in the biological processes underlying tissuespecific differentiation.

Proteins with potential functions related to signal transduction formed the largest group (55 proteins) of integral proteins identified in the plasma membrane of differentiating xylem and phloem (Table 1). Among the detected receptors, the function of most of them is yet to be investigated and only a few have been characterized for their roles in mediating signal pathways. ST263, a homolog of BRL2, was a receptor-like kinase protein detected in xylem. In Arabidopsis, BRL2 affects provascular cells differentiation and serves as an integrator of brassinosteroids (BRs) and Auxin signals with the help of its interacting proteins VIT [VH1-interacting tetratricopeptide repeat (TPR)-containing protein] and VIK (VH1-interacting kinase) (Ceserani et al. 2009; Cano-Delgado et al. 2004; Clay and Nelson 2002). ST651, a homolog of CRE1 that is a receptor histidine kinase mediating cytokinin signaling (Nieminen et al. 2008; Mahonen et al. 2000), 


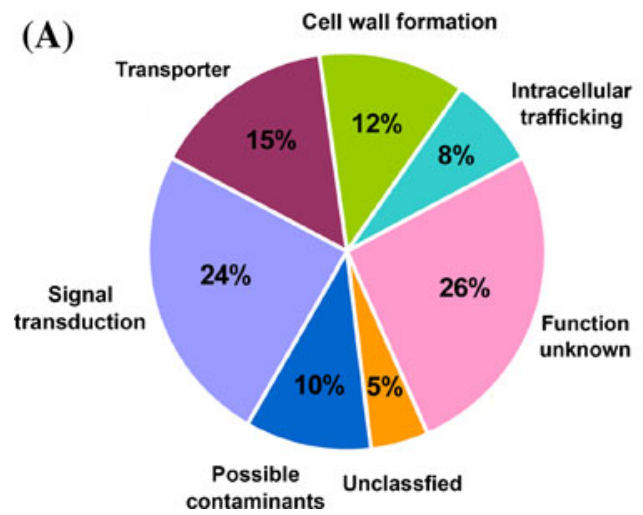

Fig. 5 Function classification of the identified plasma integral proteins from developing xylem and phloem tissue. (a) Functional classification of the identified plasma integral proteins, (b) distribution of the identified plasma integral proteins from xylem and phloem

was detected in cambium. In addition to receptor kinases, auxin-responsive family proteins, glutamate receptor, GTPbinding family proteins and other signaling proteins were also detected. In the other reported study, a total of 24 signaling proteins are identified in the xylem and cambium/ phloem (Nilsson et al. 2010), of which, 21 were also identified in our study, indicating a consistency in the detection of the signaling-related proteins between our results.

Transporters are a major class of proteins in the plasma membrane and include a variety of pumps, carriers and channels. In our study, 34 transporter proteins were identified, including aquaporins, ABC transporters, sugar transporters, cadmium-transporting ATPase, copper-transporting ATPase, $\mathrm{H}^{+}$-ATPase, and other likely transporter proteins. Aquaporins formed the largest group of transporter proteins identified in the plasma membrane. A total of 10 aquaporins proteins (TR119, TR271, TR283, TR565, TR256, TR281, TR282, TR296, TR303, TR543) were identified, of which 7 (TR271, TR283, TR256, TR281, TR282, TR296, TR303) were present in both xylem and phloem, 1 (TR119) in xylem and 2 (TR565, TR543) in phloem. This finding consistently reflects the fact that the vascular system is heavily engaged in water distribution which enables the developing xylem or phloem cells to effectively transport nutrients and photosynthetic products.

Twenty-seven integral proteins related to cell wall formation and carbohydrate metabolisms were identified, including cellulose synthases (CesAs) and other proteins known to be localized on the plasma membrane. In Populus, 18 gene loci encode 17 different CesA proteins. In the present study, a total of 10 CesAs (CW232, CW270, CW234, CW302, CW308, CW278, CW310, CW289, CW341, CW321), corresponding to CesA1-A, CesA3-A, CesA3-C, CesA3-D, CesA4, CesA6-E, CesA7-A, CesA7$\mathrm{B}$, CesA8-A and CesA8-B were detected in xylem and phloem tissues. Two endo-1,4- $\beta$-D-glucanases were

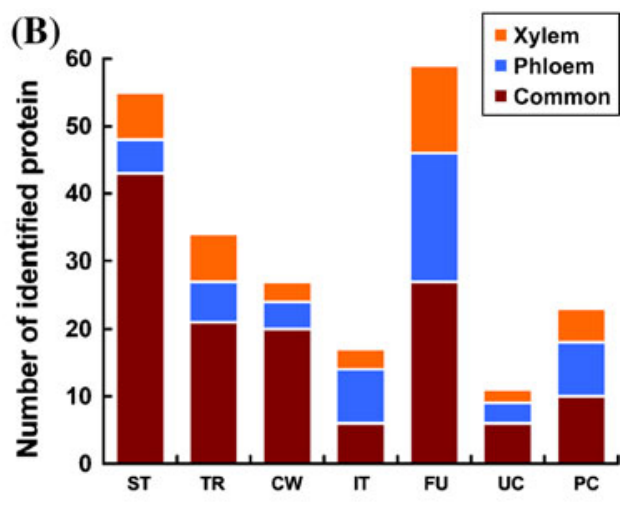

tissue. $S T$ signal transduction, $T R$ transporter, $C W$ cell wall formation, $I T$ intracellular trafficking, $F U$ function unknown, $U C$ unclassified, $P C$ possible contaminants

detected in both developing xylem and phloem tissues. One of them (CW196) is a homolog of the Arabidopsis KORRIGAN protein which is suggested to have a role in regulating cellulose crystallinity (Nicol et al. 1998; Takahashi et al. 2009). The other endo-1,4- $\beta$-D-glucanase (CW229) is a poplar homolog of Arabidopsis AtGH9B5, belonging to Arabidopsis GH9 family(Urbanowicz et al. 2007), indicating this gene may play a role in secondary xylem differentiation in poplar. A COBRA-like protein (CW18) was detected in xylem. The Cobra gene encodes an extracellular glycosyl-phosphatidyl inositol-anchored protein and its mutation results in a disordered deposition of cellulose microfibrils and cellulose synthesis reduction (Roudier et al. 2005; Schindelman et al. 2001).

A number of other proteins involved in intracellular trafficking were also detected, such as SNAREs (IT617), syntaxins (IT315, IT628, IT629, IT631) and prenylated rab acceptors(IT592, IT601). These proteins are involved in membrane trafficking for the recycling of plasma membrane proteins (Chen and Scheller 2001; Sanderfoot et al. 2001; Martincic et al. 1997; Gougeon et al. 2002). During secondary vascular development, cells undergo a rapid process of differentiation from cambium divided cells to specialized wall-thickened cells. Thus intracellular trafficking could become active as proteins on the plasma membrane turnover.

A fairly large group of the detected proteins (55 proteins) have functions which were unknown. Some of these proteins could play a role in various biological events over the course of vascular cell differentiation and cell wall formation. For example, the homolog of the DUF231 proteins (FU239 and FU448) in Arabidopsis was reported recently to be required for cellulose synthesis (Bischoff et al. 2010). The identification of these proteins, which may participate in vascular cell differentiation, presents new targets for further investigations. 
A total of 452 soluble proteins (Table S5) were detected in association with plasma membrane. A few of them are known to be associated with the plasma membrane or involved in cell wall formation. Sucrose synthase (SUSY, ID 377,378 ), which affects cellulose synthesis, has been investigated for its association with the cellulose synthase complex on the plasma membrane (Haigler et al. 2001; Amor et al. 1995; Fujii et al. 2010). Kinesin proteins (ID 675) and katanin-like proteins (ID 36) may play a role in oriented deposition of cellulose microfibrils and cell wall biosynthesis (Burk and Ye 2002; Zhong et al. 2002). However, the function as well as the association between the soluble proteins and the plasma membrane remains to be determined.

Expression of the receptor kinase genes in secondary vascular cells

The plasma proteins found in poplar secondary vascular tissues include a large group of yet to be characterized receptor-like kinases (Table 1). We are particularly interested in understanding how this group of protein are involved in intercellular communication during xylem differentiation. We analyzed their domain structures and found that the 50 proteins could be classified into 3 lectinreceptor-like kinases (Lectin-RLK), 21 leucine-rich-repeat receptor kinases (LRR-RLK), 22 receptor-like kinases (RLK), and 2 receptor histidine kinases (RHK) (Fig. 6). In order to measure the quantitative expression of these kinases in cells from the xylem, cambium, phloem and cortex, gene-specific primers (Table S6) were designed for real-time RT-PCR analysis of the 50 RLK transcripts. The expression of 46 of the 50 RLKs detected initially was confirmed in cambium meristem and differentiating cells (Fig. 7). These RLK genes displayed distinct cell-specific expression patterns. As showed in Figs. 7, 5 RLK genes were found to be specifically expressed in xylem. 12 RLK genes were expressed in cambium and xylem cells. 14 RLK genes were specifically expressed in cambium cells. 4 RLK genes were predominantly expressed in phloem cells. 11 RLK genes were expressed in cortex cells as well in xylem, phloem, or cambium cells. During differentiation from cambium meristem cells to xylem and phloem, the results showed that the RLKs were differentially expressed at various stages. However, what roles these RLKs play in intercellular communications during vascular differentiation remain yet to be investigated. As our results indicated that the process of vascular differentiation involves expression of a large group of RLK genes, the expressions of some of these RLKs are also reported in transcriptomic profiling of the secondary growth in poplar and the xylem differentiation in Arabidopsis (Dharmawardhana et al. 2010; Schrader et al. 2004; Zhao et al. 2005; Ko et al.

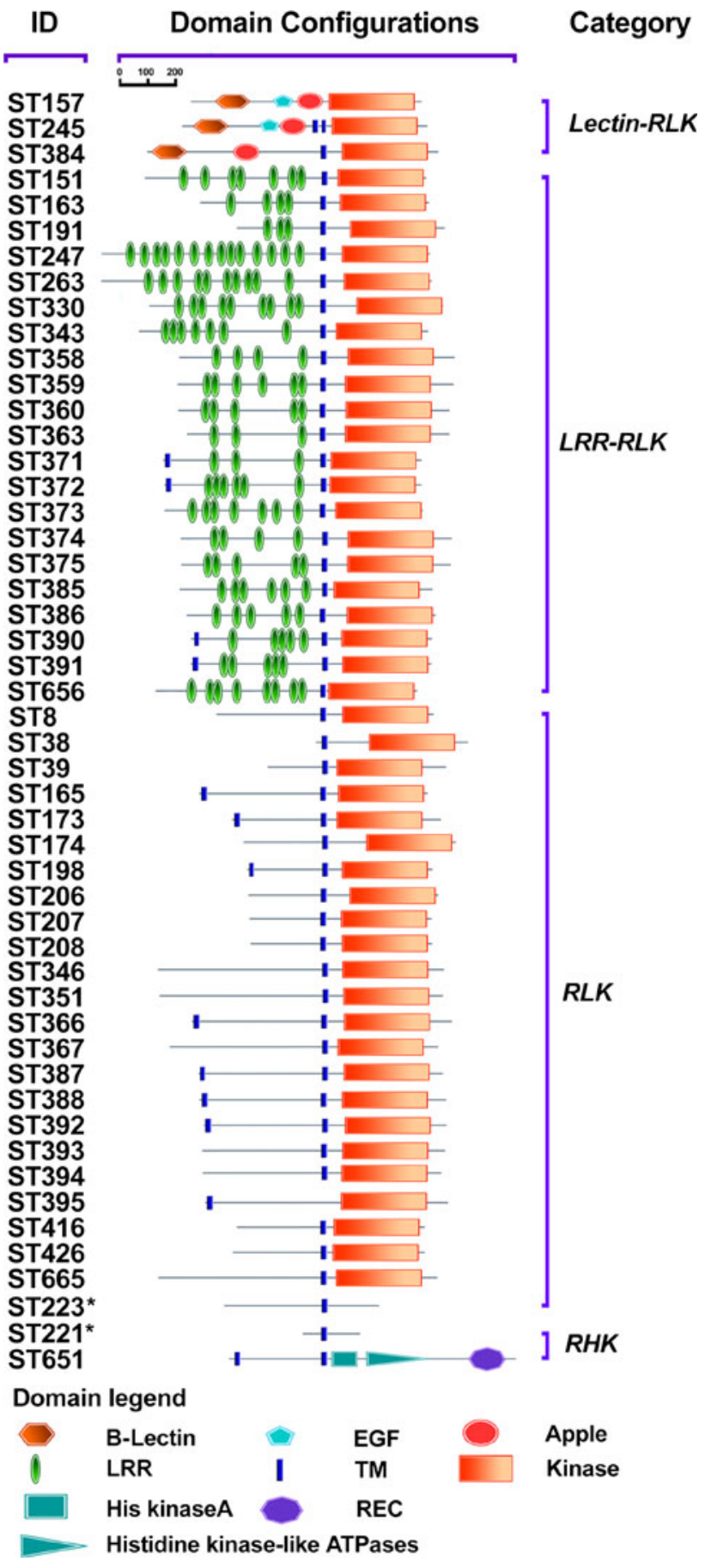

Fig. 6 Schematic structure of the RLKs identified in poplar differentiating vascular tissues. The RLKs were classified according to their domain structures. Protein domain configurations were predicted by the SMART program (http://smart.embl-heidelberg.de). Star indicates proteins with only partial sequences available

2006). For example, ST385 was found to be expressed in cambium and was also detected in the region adjacent to cambium cells in a high-resolution transcript profile study on poplar (Schrader et al. 2004). ST198 was found to be expressed in cambium and xylem, while the expression of 

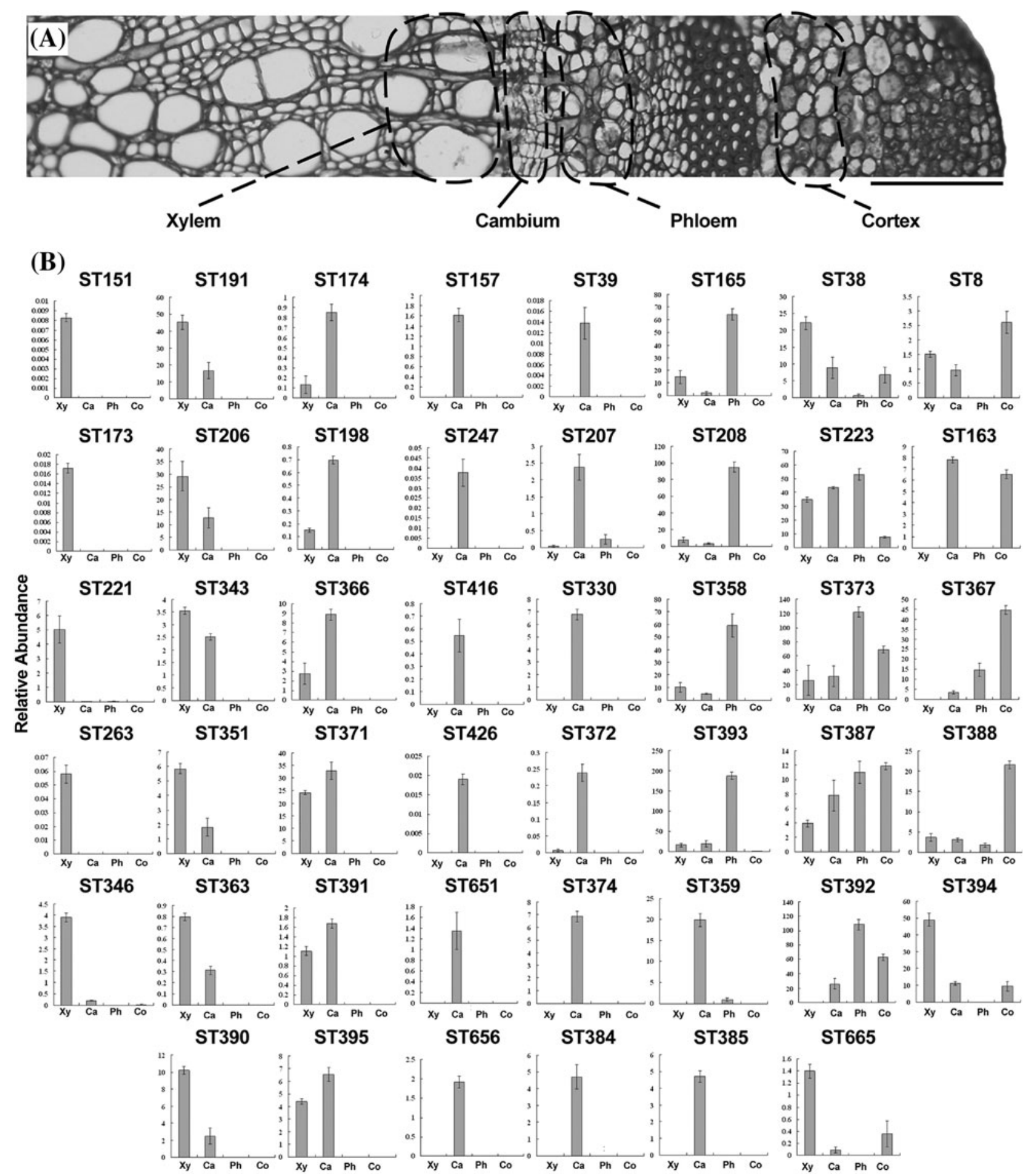

Fig. 7 Expression of receptor-like kinase genes in differentiating vascular cells. Four types of tissue cells: xylem, cambium, phloem and cortex, were collected by laser microdissection. Transcript abundance of the detected RLK genes was measured via quantitative real-time PCR analysis. (a) Transverse sections of Populus stem at the sixth internode. Samples of the collected cells were circled by broken line. Bar $100 \mu \mathrm{m}$. (b) Relative transcript abundance of the RLK genes in xylem, cambium, phloem and cortex cells. 5 RLK genes: ST151, ST173, ST221, ST263 and ST346, were specifically expressed in xylem; 12 RLK genes:

ST191, ST174, ST206, ST198, ST343, ST366, ST351, ST371, ST363, ST391, ST390 and ST395, were expressed in cambium and xylem cells; 14 RLK genes: ST157, ST39, ST246, ST207, ST416, ST330, ST426, ST372, ST651, ST374, ST359, ST656, ST384 and ST385, were specifically expressed in cambium cells; 4 RLK genes: ST165, ST208, ST358 and ST393, were predominantly expressed in phloem cells; 11 RLK genes: ST38, ST8, ST223, ST163, ST373, ST367, ST387, ST388, ST392, ST394 and ST665, were expressed in cortex cells and other cells. $X y$ xylem, $C a$ Cambium, $P h$ phloem, $C o$ cortex 
its homolog in Arabidopsis was detected in root cambium and upregulated during stem xylem differention (Zhao et al. 2005; Ko et al. 2006). ST346 and ST351 were found to be expressed mainly in xylem, while the expression of their homolog in Arabidopsis was detected in root secondary xylem and upregulated during stem xylogensis (Zhao et al. 2005; Ko et al. 2006). The expression of ST223 and ST208 which were detected in secondary phloem was found to be upregulated during the transition from primary to secondary stem development in popar (Dharmawardhana et al. 2010). The expression of ST247, ST263, ST360, ST363, ST366, ST375, ST426 was also consistent with that of their homologs in the process of Arabidopsis secondary tissue development (Zhao et al. 2005; Ko et al. 2006).

\section{Discussion}

The plasma membrane hosts a large number of proteins that are involved in a variety of cellular processes including cell-to-cell communication, cross membrane transportation, catalysis, intercellular attachment, et cetera. Membrane proteins in plants, particularly those that participate in tissue differentiation, are rarely studied. In this study, we carried out a proteomic profiling of plasma membrane from the differentiating xylem and phloem tissues of poplar. As a result, more than 1,500 proteins were detected in association with the isolated plasma membrane. Among them, a total of 226 proteins were characterized via bioinformatics as integral membrane proteins with functions mainly related to signaling, cross membrane transport, cell wall formation and carbohydrate metabolism, and intracellular trafficking. A group of proteins with unknown functions were also identified which presents potentially new targets for future studies of the plasma membrane. Recently, another study also reported the detection of a total of 956 proteins including 213 integral membrane proteins from the plasma membrane of Populus leaf, xylem and phloem (Nilsson et al. 2010). The results of the two studies showed a considerable degree of consistency in their classification of protein function and offered two independent categorizations of the integral proteins found in differentiating vascular tissues. Assessment of the two sets of independently obtained data will provide valuable information towards a better understanding of the mechanisms underlying the secondary growth process in plants, an important biological process which remains little understood.

Increases in the diameter of plant stems, a main consequence of secondary growth, depend on the activities of the secondary vascular cambium and involves a sequence of biological events including vascular cambium cell division, orientated cell differentiation, specialized cell wall thickening and programmed cell death.
A major process in cell wall thickening is cellulose synthesis, which is believed to be mediated by CesA function. CesAs in Arabidopsis are known to be divided into two types according to their involvement in primary or secondary wall formation (Desprez et al. 2007; Persson et al. 2007; Taylor et al. 2003). In poplar, recent studies have suggested that both types of CesAs are simultaneously involved in secondary wall formation (Suzuki et al. 2006; Song et al. 2010). Here, a total of 10 CesA proteins are detected in developing xylem tissue, providing additional evidence to suggest that both types of CesAs participate in secondary wall formation during poplar vascular differentiation. Detected proteins also included KORRIGAN and COBRA, which are believe to play a role in cell wall formation. Mutation in korrigan leads to a significant reduction in cellulose content and crystallinity (Nicol et al. 1998; Szyjanowicz et al. 2004; Maloney and Mansfield 2010). Overexpression of korl and its poplar homolog leads to decreased cellulose crystallinity in Arabidopsis stem (Takahashi et al. 2009). COBRA may play a role in regulating microfibril orientation and deposition (Roudier et al. 2005; Schindelman et al. 2001). Though the mechanisms of how these proteins regulate the cell wall formation are still not fully understood, our results here again confirmed that KOR1 and COBRA are localized in the plasma membrane.

Cell-to-cell communication plays a crucial role in determining cell fate and differentiation, especially for immobile plant cells. In the present study, a group of RLKs were identified in the differentiating xylem and phloem, suggesting their involvement in secondary vascular cambium differentiation. Generally, ligand-RLK signaling is believed as a crucial pathway regulating cell differentiation in plants (De Smet et al. 2009). Thus, the identification of a group of RLKs which may play a role in secondary vascular differentiation is of particular interest. It is know that the Arabidopsis genome contains more than 600 RLK genes (Shiu and Bleecker 2001). In our study, 50 RLK proteins were detected specifically in the plasma membrane of secondary differentiating tissues of poplar. Among them, 46 genes are further confirmed to be expressed in the cambium which differentiates into xylem and phloem cells. However, only 2 of these RLK genes, ST263 and ST651, have been studied for their function in Arabidopsis. ST263 is a homolog of VH1/AtBRL2, which is reported to mediate brassinosteroids (BRs) and Auxin signaling and play a role in vascular differentiation in Arabidopsis (Ceserani et al. 2009; Cano-Delgado et al. 2004; Clay and Nelson 2002). During secondary growth, the ST263 gene is found to be expressed in xylem cells, suggesting that brassinosteroids (BRs) and auxin signaling also play a role in secondary vascular differentiation. ST651 is a homolog of CRE1 which is a receptor histidine kinase mediating 
cytokinin signaling and is involved in many cellular processes, including cambial development in Arabidopsis, poplar and birch (Nieminen et al. 2008; Mahonen et al. 2000). The ST651 gene was expressed specifically in cambium cells, strongly suggesting that cytokinin signaling plays an important role in cambium cell division during secondary growth.

In addition, homologs of several RLK genes were found to be specifically regulated by xylem differentiation. For example, the ST346 and ST351 genes were found to be expressed in the xylem and cambium of poplar plants used in our study. Their homolog in Arabidopsis Attg79620 is specifically expressed in xylem and induced by a vessel regulator VND6 (Zhao et al. 2005; Ko et al. 2006; OhashiIto et al. 2010). Also the ST198 gene, which was found to be expressed in xylem and cambium cells in poplar and its Arabidopsis homolog, At5g58300, is directly regulated by transcription factor VND6. (Zhao et al. 2005; Ko et al. 2006; Ohashi-Ito et al. 2010). The results here would help in the construction of yet to be characterized signaling networks which play important roles in regulating xylem (vessel cell) differentiation in poplar.

On the other hand, an Arabidopsis LRR receptor kinase, PXY/TDR and its ligand CLE41/TDIF peptide, have been recently reported play a key role in xylem-phloem patterning through controlling procambial cell division in a non-cell-autonomous manner (Hirakawa et al. 2008; Hirakawa et al. 2010; Etchells and Turner 2010). In the Populus genome, the gw1.29.276.1 gene model is the closest homolog of PXY (At5g61480) with a sequence homology of $77 \%$. However, neither our study nor the other study (Nilsson et al. 2010) detected a unique peptide which matches the Populus PXY homolog. The reason may be the protein identification in the two studies was unable to fully include all possible membrane proteins due to limitation of the proteomic analysis (Garbis et al. 2005). Meanwhile, whether the mechanism of the xylem-phloem patterning regulated by PXY is the same in poplar secondary growth remain to be confirmed.

Different from primary growth that is derived from apical meristems, the process of secondary growth occurs through the activities of the secondary vascular meristem. After the first tree genome was sequenced (Tuskan et al. 2006), experimental attempts to uncover the basic biological networks underlying secondary growth has yielded interesting insights into this biological process (Du and Groover 2010). The tree genome also provided a valuable database to enable an understanding of the secondary growth of trees at a proteomic level. Tissue-specific proteins profiles and even more specifically, the identification of subcellular proteins are among the key information required for the characterization of secondary growth. In this study, the identification of tissue-specific plasma membrane proteins as well as cell types which specifically expressed RLKs may serve as a first step to further dissect how secondary growth could have developed through various biological activities which originated from the plasma membrane.

Acknowledgments We thank Profs. Jisheng Shi, Liwei Yang (Nanjing Forestry University) and fellow lab members for their assistance in the sample collection; Dr. Jun Yao (Fudan University) for helping with the protein LC-MS/MS analysis; Dr. Weihua Tang (Institute of Plant Physiology and Ecology) for Laser microdissection. This research was supported by the National Natural Science Foundation of China (grant number 30725025), Chinese Academy of Science (grant number, KSCX2-YW-N-070) and the Ministry of Science and Technology (2009AA10Z101) to L. L.

Open Access This article is distributed under the terms of the Creative Commons Attribution Noncommercial License which permits any noncommercial use, distribution, and reproduction in any medium, provided the original author(s) and source are credited.

\section{References}

Allona I, Quinn M, Shoop E, Swope K, St Cyr S, Carlis J, Riedl J, Retzel E, Campbell MM, Sederoff R, Whetten RW (1998) Analysis of xylem formation in pine by cDNA sequencing. Proc Natl Acad Sci USA 95(16):9693-9698

Amor Y, Haigler CH, Johnson S, Wainscott M, Delmer DP (1995) A membrane-associated form of sucrose synthase and its potential role in synthesis of cellulose and callose in plants. Proc Natl Acad Sci USA 92(20):9353-9357

Baginsky S (2009) Plant proteomics: concepts, applications, and novel strategies for data interpretation. Mass Spectrom Rev 28(1):93-120

Bessueille L, Sindt N, Guichardant M, Djerbi S, Teeri TT, Bulone V (2009) Plasma membrane microdomains from hybrid aspen cells are involved in cell wall polysaccharide biosynthesis. Biochem J 420(1):93-103

Bischoff V, Nita S, Neumetzler L, Schindelasch D, Urbain A, Eshed R, Persson S, Delmer D, Scheible WR (2010) TRICHOME BIREFRINGENCE and its homolog AT5G01360 encode plantspecific DUF231 proteins required for cellulose biosynthesis in Arabidopsis. Plant Physiol 153(2):590-602

Burk DH, Ye ZH (2002) Alteration of oriented deposition of cellulose microfibrils by mutation of a katanin-like microtubule-severing protein. Plant Cell 14(9):2145-2160

Cano-Delgado A, Yin Y, Yu C, Vafeados D, Mora-Garcia S, Cheng JC, Nam KH, Li J, Chory J (2004) BRL1 and BRL3 are novel brassinosteroid receptors that function in vascular differentiation in Arabidopsis. Development 131(21):5341-5351

Ceserani T, Trofka A, Gandotra N, Nelson T (2009) VH1/BRL2 receptor-like kinase interacts with vascular-specific adaptor proteins VIT and VIK to influence leaf venation. Plant J 57(6): $1000-1014$

Chen Y, Scheller R (2001) SNARE-mediated membrane fusion. Nat Rev Mol Cell Biol 2(2):98-106

Clay N, Nelson T (2002) VH1, a provascular cell-specific receptor kinase that influences leaf cell patterns in Arabidopsis. The Plant Cell Online 14(11):2707-2722

De Smet I, Voss U, Jurgens G, Beeckman T (2009) Receptor-like kinases shape the plant. Nat Cell Biol 11(10):1166-1173 
Desprez T, Juraniec M, Crowell EF, Jouy H, Pochylova Z, Parcy F, Hofte H, Gonneau M, Vernhettes S (2007) Organization of cellulose synthase complexes involved in primary cell wall synthesis in Arabidopsis thaliana. Proc Natl Acad Sci USA 104(39):15572-15577

Dharmawardhana P, Brunner AM, Strauss SH (2010) Genome-wide transcriptome analysis of the transition from primary to secondary stem development in Populus trichocarpa. BMC Genomics $11: 150$

$\mathrm{Du}$ J, Groover A (2010) Transcriptional regulation of secondary growth and wood formation. J Integr Plant Biol 52(1):17-27

Du J, Xie HL, Zhang DQ, He XQ, Wang MJ, Li YZ, Cui KM, Lu MZ (2006) Regeneration of the secondary vascular system in poplar as a novel system to investigate gene expression by a proteomic approach. Proteomics 6(3):881-895

Etchells JP, Turner SR (2010) The PXY-CLE41 receptor ligand pair defines a multifunctional pathway that controls the rate and orientation of vascular cell division. Development 137(5):767-774

Fiorani Celedon PA, de Andrade A, Meireles KG, Gallo de Carvalho MC, Caldas DG, Moon DH, Carneiro RT, Franceschini LM, Oda $S$, Labate CA (2007) Proteomic analysis of the cambial region in juvenile Eucalyptus grandis at three ages. Proteomics 7(13): 2258-2274

Fisher K, Turner S (2007) PXY, a receptor-like kinase essential for maintaining polarity during plant vascular-tissue development. Curr Biol 17(12):1061-1066

Fletcher JC, Brand U, Running MP, Simon R, Meyerowitz EM (1999) Signaling of cell fate decisions by CLAVATA3 in Arabidopsis shoot meristems. Science 283(5409):1911-1914

Fujii S, Hayashi T, Mizuno K (2010) Sucrose synthase is an integral component of the cellulose synthesis machinery. Plant Cell Physiol 51(2):294-301

Fukuda H (2004) Signals that control plant vascular cell differentiation. Nat Rev Mol Cell Biol 5(5):379-391

Garbis S, Lubec G, Fountoulakis M (2005) Limitations of current proteomics technologies. J Chromatogr A 1077(1):1-18

Gougeon PY, Prosser DC, Da-Silva LF, Ngsee JK (2002) Disruption of Golgi morphology and trafficking in cells expressing mutant prenylated rab acceptor-1. J Biol Chem 277(39):36408-36414

Haigler CH, Ivanova-Datcheva M, Hogan PS, Salnikov VV, Hwang S, Martin K, Delmer DP (2001) Carbon partitioning to cellulose synthesis. Plant Mol Biol 47(1-2):29-51

Hertzberg M, Aspeborg H, Schrader J, Andersson A, Erlandsson R, Blomqvist K, Bhalerao R, Uhlen M, Teeri TT, Lundeberg J, Sundberg B, Nilsson P, Sandberg G (2001) A transcriptional roadmap to wood formation. Proc Natl Acad Sci USA 98(25): 14732-14737

Hirakawa Y, Shinohara H, Kondo Y, Inoue A, Nakanomyo I, Ogawa M, Sawa S, Ohashi-Ito K, Matsubayashi Y, Fukuda H (2008) Non-cell-autonomous control of vascular stem cell fate by a CLE peptide/receptor system. Proc Natl Acad Sci USA 105(39): $15208-15213$

Hirakawa Y, Kondo Y, Fukuda H (2010) TDIF peptide signaling regulates vascular stem cell proliferation via the WOX4 homeobox gene in Arabidopsis. Plant Cell 22(8):2618-2629

Hurkman WJ, Tanaka CK (1986) Solubilization of Plant Membrane Proteins for Analysis by Two-Dimensional Gel Electrophoresis. Plant Physiol 81(3):802-806

Isaacson T, Damasceno CM, Saravanan RS, He Y, Catala C, Saladie M, Rose JK (2006) Sample extraction techniques for enhanced proteomic analysis of plant tissues. Nat Protoc 1(2):769-774

Keller A, Nesvizhskii AI, Kolker E, Aebersold R (2002) Empirical statistical model to estimate the accuracy of peptide identifications made by MS/MS and database search. Anal Chem 74(20): 5383-5392
Ko JH, Beers EP, Han KH (2006) Global comparative transcriptome analysis identifies gene network regulating secondary xylem development in Arabidopsis thaliana. Mol Gen Genomics 276(6):517-531

Komatsu S, Konishi H, Hashimoto M (2007) The proteomics of plant cell membranes. J Exp Bot 58(1):103-112

Lenhard M, Laux T (1999) Shoot meristem formation and maintenance. Curr Opin Plant Biol 2(1):44-50

Lilley KS, Dupree P (2007) Plant organelle proteomics. Curr Opin Plant Biol 10(6):594-599

Mahonen AP, Bonke M, Kauppinen L, Riikonen M, Benfey PN, Helariutta Y (2000) A novel two-component hybrid molecule regulates vascular morphogenesis of the Arabidopsis root. Genes Dev 14(23):2938-2943

Maloney VJ, Mansfield SD (2010) Characterization and varied expression of a membrane-bound endo-beta-1, 4-glucanase in hybrid poplar. Plant Biotechnol J 8(3):294-307

Martincic I, Peralta ME, Ngsee JK (1997) Isolation and characterization of a dual prenylated Rab and VAMP2 receptor. J Biol Chem 272(43):26991-26998

Nesvizhskii AI, Keller A, Kolker E, Aebersold R (2003) A statistical model for identifying proteins by tandem mass spectrometry. Anal Chem 75(17):4646-4658

Nicol F, His I, Jauneau A, Vernhettes S, Canut H, Hofte H (1998) A plasma membrane-bound putative endo-1, 4-beta-D-glucanase is required for normal wall assembly and cell elongation in Arabidopsis. EMBO J 17(19):5563-5576

Nieminen K, Immanen J, Laxell M, Kauppinen L, Tarkowski P, Dolezal K, Tahtiharju S, Elo A, Decourteix M, Ljung K, Bhalerao R, Keinonen K, Albert VA, Helariutta Y (2008) Cytokinin signaling regulates cambial development in poplar. Proc Natl Acad Sci USA 105(50):20032-20037

Nilsson R, Bernfur K, Gustavsson N, Bygdell J, Wingsle G, Larsson C (2010) Proteomics of plasma membranes from poplar trees reveals tissue distribution of transporters, receptors, and proteins in cell wall formation. Mol Cell Proteomics 9(2):368-387

Nohzadeh Malakshah S, Habibi Rezaei M, Heidari M, Salekdeh GH (2007) Proteomics reveals new salt responsive proteins associated with rice plasma membrane. Biosci Biotechnol Biochem 71(9):2144-2154

Ohashi-Ito K, Oda Y, Fukuda H (2010) Arabidopsis VASCULARRELATED NAC-DOMAIN6 directly regulates the genes that govern programmed cell death and secondary wall formation during xylem differentiation. Plant Cell 22(10):3461-3473

Pavy N, Boyle B, Nelson C, Paule C, Giguere I, Caron S, Parsons LS, Dallaire N, Bedon F, Berube H, Cooke J, Mackay J (2008) Identification of conserved core xylem gene sets: conifer cDNA microarray development, transcript profiling and computational analyses. New Phytol 180(4):766-786

Persson S, Paredez A, Carroll A, Palsdottir H, Doblin M, Poindexter P, Khitrov N, Auer M, Somerville CR (2007) Genetic evidence for three unique components in primary cell-wall cellulose synthase complexes in Arabidopsis. Proc Natl Acad Sci USA 104(39):15566-15571

Reiland S, Messerli G, Baerenfaller K, Gerrits B, Endler A, Grossmann J, Gruissem W, Baginsky S (2009) Large-scale Arabidopsis phosphoproteome profiling reveals novel chloroplast kinase substrates and phosphorylation networks. Plant Physiol 150(2):889-903

Roudier F, Fernandez AG, Fujita M, Himmelspach R, Borner GH, Schindelman G, Song S, Baskin TI, Dupree P, Wasteneys GO, Benfey PN (2005) COBRA, an Arabidopsis extracellular glycosyl-phosphatidyl inositol-anchored protein, specifically controls highly anisotropic expansion through its involvement in cellulose microfibril orientation. Plant Cell 17(6):1749-1763 
Sanderfoot AA, Kovaleva V, Bassham DC, Raikhel NV (2001) Interactions between syntaxins identify at least five SNARE complexes within the Golgi/prevacuolar system of the Arabidopsis cell. Mol Biol Cell 12(12):3733-3743

Sandstrom RP, Deboer AH, Lomax TL, Cleland RE (1987) Latency of Plasma Membrane H-ATPase in vesicles isolated by aqueous phase partitioning: increased substrate accessibility or enzyme activation. Plant Physiol 85(3):693-698

Santoni V, Rouquie D, Doumas P, Mansion M, Boutry M, Degand H, Dupree P, Packman L, Sherrier J, Prime T, Bauw G, Posada E, Rouze P, Dehais P, Sahnoun I, Barlier I, Rossignol M (1998) Use of a proteome strategy for tagging proteins present at the plasma membrane. Plant J 16(5):633-641

Saravanan RS, Rose JK (2004) A critical evaluation of sample extraction techniques for enhanced proteomic analysis of recalcitrant plant tissues. Proteomics 4(9):2522-2532

Schindelman G, Morikami A, Jung J, Baskin TI, Carpita NC, Derbyshire P, McCann MC, Benfey PN (2001) COBRA encodes a putative GPI-anchored protein, which is polarly localized and necessary for oriented cell expansion in Arabidopsis. Genes Dev 15(9):1115-1127

Schindler J, Nothwang HG (2006) Aqueous polymer two-phase systems: effective tools for plasma membrane proteomics. Proteomics 6(20):5409-5417

Schrader J, Nilsson J, Mellerowicz E, Berglund A, Nilsson P, Hertzberg M, Sandberg G (2004) A high-resolution transcript profile across the wood-forming meristem of poplar identifies potential regulators of cambial stem cell identity. Plant Cell 16(9):2278-2292

Shiu SH, Bleecker AB (2001) Plant receptor-like kinase gene family: diversity, function, and signaling. Sci STKE 2001 (113):re22

Song D, Shen J, Li L (2010) Characterization of cellulose synthase complexes in Populus xylem differentiation. New Phytol 187: $777-790$

Suzuki S, Li L, Sun YH, Chiang VL (2006) The cellulose synthase gene superfamily and biochemical functions of xylem-specific cellulose synthase-like genes in Populus trichocarpa. Plant Physiol 142(3):1233-1245

Sze $\mathrm{H}(1985) \mathrm{H}^{+}$-translocating atpases-advances using membranevesicles. Annu Rev Plant Physiol Plant Mol Biol 36:175-208

Szyjanowicz PM, McKinnon I, Taylor NG, Gardiner J, Jarvis MC, Turner SR (2004) The irregular xylem 2 mutant is an allele of korrigan that affects the secondary cell wall of Arabidopsis thaliana. Plant J 37(5):730-740

Takahashi J, Rudsander UJ, Hedenstrom M, Banasiak A, Harholt J, Amelot N, Immerzeel P, Ryden P, Endo S, Ibatullin FM, Brumer H, del Campillo E, Master ER, Scheller HV, Sundberg B, Teeri TT, Mellerowicz EJ (2009) KORRIGAN1 and its aspen homolog PttCel9A1 decrease cellulose crystallinity in Arabidopsis stems. Plant Cell Physiol 50(6):1099-1115

Tan S, Tan HT, Chung MC (2008) Membrane proteins and membrane proteomics. Proteomics 8(19):3924-3932
Tanaka N, Fujita M, Handa H, Murayama S, Uemura M, Kawamura Y, Mitsui T, Mikami S, Tozawa Y, Yoshinaga T, Komatsu S (2004) Proteomics of the rice cell: systematic identification of the protein populations in subcellular compartments. Mol Genet Genomics 271(5):566-576

Taylor NG, Howells RM, Huttly AK, Vickers K, Turner SR (2003) Interactions among three distinct CesA proteins essential for cellulose synthesis. Proc Natl Acad Sci USA 100(3):1450-1455

Tuskan GA, Difazio S, Jansson S, Bohlmann J, Grigoriev I, Hellsten U, Putnam N, Ralph S, Rombauts S, Salamov A, Schein J, Sterck L, Aerts A, Bhalerao RR, Bhalerao RP, Blaudez D, Boerjan W, Brun A, Brunner A, Busov V, Campbell M, Carlson J, Chalot M, Chapman J, Chen GL, Cooper D, Coutinho PM, Couturier J, Covert S, Cronk Q, Cunningham R, Davis J, Degroeve S, Dejardin A, Depamphilis C, Detter J, Dirks B, Dubchak I, Duplessis S, Ehlting J, Ellis B, Gendler K, Goodstein D, Gribskov M, Grimwood J, Groover A, Gunter L, Hamberger B, Heinze B, Helariutta Y, Henrissat B, Holligan D, Holt R, Huang $\mathrm{W}$, Islam-Faridi $\mathrm{N}$, Jones $\mathrm{S}$, Jones-Rhoades $\mathrm{M}$, Jorgensen $\mathrm{R}$, Joshi C, Kangasjarvi J, Karlsson J, Kelleher C, Kirkpatrick R, Kirst M, Kohler A, Kalluri U, Larimer F, Leebens-Mack J, Leple JC, Locascio P, Lou Y, Lucas S, Martin F, Montanini B, Napoli C, Nelson DR, Nelson C, Nieminen K, Nilsson O, Pereda V, Peter G, Philippe R, Pilate G, Poliakov A, Razumovskaya J, Richardson P, Rinaldi C, Ritland K, Rouze P, Ryaboy D, Schmutz J, Schrader J, Segerman B, Shin H, Siddiqui A, Sterky F, Terry A, Tsai CJ, Uberbacher E, Unneberg P, Vahala J, Wall K, Wessler S, Yang G, Yin T, Douglas C, Marra M, Sandberg G, Van de Peer Y, Rokhsar D (2006) The genome of black cottonwood, Populus trichocarpa (Torr. \& Gray). Science 313 (5793):1596-1604

Urbanowicz BR, Bennett AB, Del Campillo E, Catala C, Hayashi T, Henrissat B, Hofte H, McQueen-Mason SJ, Patterson SE, Shoseyov O, Teeri TT, Rose JK (2007) Structural organization and a standardized nomenclature for plant endo-1, 4-betaglucanases (cellulases) of glycosyl hydrolase family 9. Plant Physiol 144(4):1693-1696

Yu QB, Li G, Wang G, Sun JC, Wang PC, Wang C, Mi HL, Ma WM, Cui J, Cui YL, Chong K, Li YX, Li YH, Zhao Z, Shi TL, Yang $\mathrm{ZN}$ (2008) Construction of a chloroplast protein interaction network and functional mining of photosynthetic proteins in Arabidopsis thaliana. Cell Res 18(10):1007-1019

Zhang M, Li G, Huang W, Bi T, Chen G, Tang Z, Su W, Sun W (2010) Proteomic study of Carissa spinarum in response to combined heat and drought stress. Proteomics 10:3117-3129

Zhao CS, Craig JC, Petzold HE, Dickerman AW, Beers EP (2005) The xylem and phloem transcriptomes from secondary tissues of the Arabidopsis root-hypocotyl. Plant Physiol 138(2):803-818

Zhong R, Burk DH, Morrison WH 3rd, Ye ZH (2002) A kinesin-like protein is essential for oriented deposition of cellulose microfibrils and cell wall strength. Plant Cell 14(12):3101-3117 